\title{
Spectrally smooth and spatially uniform sound radiation from a thin plate structure using band gaps
}

Jung, Jaesoon; Jeong, Cheol-Ho; Jensen, Jakob Søndergaard

Published in:

Journal of Sound and Vibration

Link to article, DOI:

10.1016/j.jsv.2020.115187

Publication date:

2020

Document Version

Peer reviewed version

Link back to DTU Orbit

Citation (APA):

Jung, J., Jeong, C-H., \& Jensen, J. S. (2020). Spectrally smooth and spatially uniform sound radiation from a thin plate structure using band gaps. Journal of Sound and Vibration, 471, [115187].

https://doi.org/10.1016/j.jsv.2020.115187

\section{General rights}

Copyright and moral rights for the publications made accessible in the public portal are retained by the authors and/or other copyright owners and it is a condition of accessing publications that users recognise and abide by the legal requirements associated with these rights.

- Users may download and print one copy of any publication from the public portal for the purpose of private study or research.

- You may not further distribute the material or use it for any profit-making activity or commercial gain

- You may freely distribute the URL identifying the publication in the public portal

If you believe that this document breaches copyright please contact us providing details, and we will remove access to the work immediately and investigate your claim. 


\title{
Spectrally smooth and spatially uniform sound radiation from a thin plate structure using band gaps
}

\author{
Jaesoon Jung $^{1 *}$, Cheol-Ho Jeong ${ }^{1}$, Jakob S. Jensen ${ }^{2}$
}

1: Department of Electrical Engineering, Technical University of Denmark, $2800 \mathrm{Kgs}$.

Lyngby, Denmark

2: Department of Mechanical Engineering, Technical University of Denmark, $2800 \mathrm{Kgs}$.

Lyngby, Denmark

Submitted to Journal of Sound and Vibration

Submitted: September 19, 2019

Includes 18 figures

* Corresponding author

Postal address: Acoustic Technology, Department of Electrical Engineering, Technical

University of Denmark, Ørsteds Plads, 2800, Kgs. Lyngby, Denmark

E-mail address: jjung@elektro.dtu.dk 


\begin{abstract}
As an alternative to conventional loudspeakers, panel loudspeakers have been investigated. However, it is challenging to avoid structural modes in a panel loudspeaker, which results in an uneven frequency spectrum and highly directional sound radiation. Here, we present a technique to eliminate modes in a frequency range of interest based on a band gap (BG) structure that forbids the propagation of waves. In order to open a BG between 300 and 500 $\mathrm{Hz}$, a thin aluminum plate with periodic resonators is considered. Using finite element (FE) simulations, vibro-acoustic responses of the BG structure are analyzed, leading to more spectrally smooth and spatially uniform sound radiation. Experimental results agree well with the numerical predictions, displaying a smoother frequency spectrum and smaller variation of sound pressure at six different measurement orientations in an anechoic chamber. For this specific case, the overall SPL is amplified by $5.5 \mathrm{~dB}$ and the standard deviation is reduced by $5.7 \mathrm{~dB}$ at frequencies ranging from $300 \mathrm{~Hz}$ to $500 \mathrm{~Hz}$.
\end{abstract}

Keyword: Band gap; Flat panel loudspeaker; Sound radiation; Vibro-acoustics; 


\section{Introduction}

Loudspeakers are essential components for many modern digital devices to transfer information, play music, and even reduce noise with active control. Despite the importance of the loudspeaker, it is usually challenging to install a loudspeaker driver into a limited space, so one needs a strategy to install loudspeakers compactly [1]. To name a few examples, TV displays are too thin to insert a speaker driver, and subwoofers in an automobile audio system increase the total weight while reducing the available space $[2,3]$.

Thus, the idea that a surface of mechanical structures can serve as a loudspeaker itself is appealing. In this regard, flat panel loudspeakers (FPSs) [4,5] which produce sound using a flat and thin panel excited by several actuators have attracted engineers to add an acoustic function for devices where allowable spaces are limited, e.g. for display panels [2,6] and in automobiles $[3,7]$. In contrast to the conventional loudspeaker drivers, the FPS uses the dense modes of a thin panel to generate sound. Hence, at high frequencies where the modal density is high, the FPS produces a spectrally smooth frequency response [4]. Conversely, at low frequencies where the modes are sparse, the FPS usually produces an uneven frequency spectrum and directive sound radiation [8]. Besides, at low frequencies, it is difficult to localize the sound source on the surface because the entire surface vibrates simultaneously, whereas the vibration is confined around the positions of actuators at high frequencies due to a high modal density [9]. These low frequency characteristics are drawbacks of the FPS since its initial development $[2]$.

Considerable research interest has been given to remedy these problems. Passive design methods were developed, extending the optimization approaches applied to conventional loudspeakers [10,11], e.g., determining actuator placement using numerical simulations and optimization algorithms [12-14], and exploiting orthotropic materials to rebuild mode distributions $[15,16]$. Additionally, vibration control methods tackled the problem by means of signal processing, e.g. the modal crossover network, which selectively excites specific modes by balancing modal forces [17], and shaping the vibration profile using active vibration control based on an array of actuators [3,7,18-21]. These previous works mainly focused on determining the excitation and mode distributions of a panel structure, but it is still challenging to solve the fundamental issue of sparse modes at low frequencies.

We present a mode elimination technique using a band gap (BG) structure, which forbids the propagation of bending waves within a frequency band called a band gap (or also called stop band) [22-25]. Usually, BG structures consist of a periodic arrangement of elements such 
as scatterers [26], resonators [27], and with use of inertial amplification [28], which make wavenumber imaginary within a BG range. Due to the imaginary wavenumber, bending waves decay exponentially and become unlikely to reflect from the boundaries; thus, the BG structure allows no standing waves and no modes within the BG [26]. Fig. 1 schematically illustrates the mode elimination technique and wave propagation in a BG structure in which the evanescent wave decays before it reaches boundaries [29-31] in comparison to a bare plate. In the figure, the green squares represent the periodic elements that compose the BG structure.

In this paper, we aim to produce a spectrally smooth and spatially uniform sound radiation at low frequencies. The target frequency band is chosen to range from $300 \mathrm{~Hz}$ to $500 \mathrm{~Hz}$ for two reasons, one being that many conventional FPSs suffer from uneven sound radiation characteristics due to sparse modes in this frequency range [12-17], and the second being that many small loudspeakers in small mobile devices cannot radiate sound well in this frequency range. Note that even lower frequency ranges can be targeted by designing a BG structure [27]. To create a BG in the target frequency range, we consider a periodic arrangement of resonators on a thin panel structure. The finite structure with periodic resonators can eliminate modes within the BG range, thus confining vibrations near the position of the actuator [29]. These two features of the BG structure smoothen the frequency spectrum and produce a spatially uniform sound radiation. For analyses of the BG structure, a finite element (FE) vibro-acoustic model is developed based on the Mindlin plate theory [32]. Using the FE model, the modal characteristics and vibro-acoustic responses of the BG structure are investigated. The numerical results are validated with experiments by measuring vibration fields and the sound pressure radiated from the BG structure.

This work is structured as follows: we present the theories of the BG and vibro-acoustic modelling in Section 2. The numerical results using the developed FE model are presented in Section 3. In Section 4, experimental results are presented to validate the numerical results. Finally, Section 5 concludes this paper. 


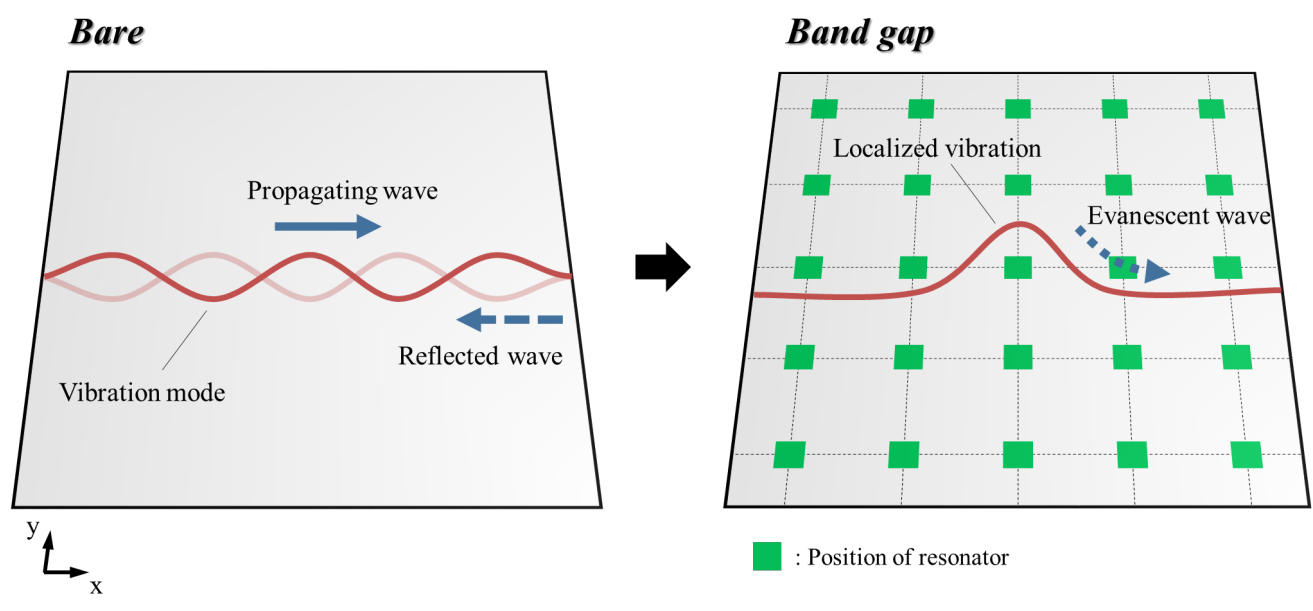

Fig. 1. Description of the mode elimination technique.

\section{Theory}

\subsection{Unit cell modelling}

Consider a periodic unit cell structure, which consists of a thin plate and a simple massspring resonator, as described in Fig. 2. Assuming a harmonic motion, the free vibration of the plate can be described with the Mindlin plate theory and the principle of virtual work as [32],

$$
-\omega^{2} \int_{S}\left[\rho h \delta w w+\frac{h^{3}}{12}\left(\delta \beta_{x} \beta_{x}+\delta \beta_{y} \beta_{y}\right)\right] \mathrm{d} S+\int_{S}\left[\delta \mathbf{\kappa}^{T} \mathbf{C}_{b} \mathbf{\kappa}+\delta \gamma^{T} \mathbf{C}_{s} \gamma\right] \mathrm{d} S=0,
$$

where $\omega$ is the angular frequency, $\rho$ is the mass density, $h$ is the thickness of the plate, $S$ is the surface area, and $\delta$ represents the virtual variables. The variables $\beta$ and $w$ are the rotational and vertical displacements, $\boldsymbol{\kappa}$ and $\boldsymbol{\gamma}$ indicate the curvature vector and the shear strain vector, respectively, defined as follows,

$$
\begin{gathered}
\boldsymbol{\kappa}=\left[\begin{array}{c}
\partial \beta_{x} / \partial x \\
\partial \beta_{y} / \partial y \\
\partial \beta_{x} / \partial y+\partial \beta_{y} / \partial x
\end{array}\right], \\
\boldsymbol{\gamma}=\left[\begin{array}{c}
\partial w / \partial x-\beta_{x} \\
\partial w / \partial y-\beta_{y}
\end{array}\right] .
\end{gathered}
$$

The matrices $\mathbf{C}_{\mathrm{b}}$ and $\mathbf{C}_{\mathrm{s}}$ in Eq. (1) are the constitutive matrices defined as,

$$
\mathbf{C}_{b}=\frac{E h^{3}}{12}\left[\begin{array}{ccc}
1 & v & 0 \\
v & 1 & 0 \\
0 & 0 & (1-v) / 2
\end{array}\right]
$$




$$
\mathbf{C}_{s}=\frac{E h k}{2(1+v)}\left[\begin{array}{ll}
1 & 0 \\
0 & 1
\end{array}\right],
$$

where $E$ is the elastic modulus, $v$ is the Poisson's ratio, and $k(=5 / 6)$ is the shear correction factor. Furthermore, the equation of motion of the mass-spring resonator is expressed as,

$$
-\omega^{2} m_{r} w_{r}+k_{r}\left(w_{r}-w_{p}\right)=f_{r p},
$$

where $m_{r}$ and $k_{r}$ are the lumped mass and stiffness of the resonator, $w_{r}$ and $w_{p}$ are the vertical displacements of the resonator and plate at the connection point, respectively.

Employing the finite element (FE) method, Eq. (1) can be discretized into a matrix equation as [32],

$$
\left(-\omega^{2} \mathbf{M}+\mathbf{K}\right) \mathbf{u}=\mathbf{0}
$$

where $\mathbf{M}$ and $\mathbf{K}$ are the mass and stiffness matrices, respectively, and $\mathbf{u}$ is the degrees of freedom (dof) vector. For the formation of FE matrices, we employ the mixed integral tensorial component (MITC) scheme to prevent the shear locking problem arising in the numerical integration of the shear stiffness term [33].

Eq. (6) can be combined with Eq. (5) by coupling the dofs at the connection point, which results in the coupled matrix equation as follows,

$$
\begin{aligned}
& \left(-\omega^{2} \tilde{\mathbf{M}}+\tilde{\mathbf{K}}\right) \tilde{\mathbf{u}}=\mathbf{0}, \\
& \tilde{\mathrm{K}}_{p p}=\mathrm{K}_{p p}+k_{r}, \\
& \tilde{\mathbf{K}}_{r r}=k_{r}, \\
& \tilde{\mathrm{K}}_{r p}=\tilde{\mathbf{K}}_{p r}=-k_{r}, \\
& \tilde{\mathbf{M}}_{r r}=m_{r},
\end{aligned}
$$

where the tilde symbol over the matrices represents the coupling between the FE matrices and the equation of motion of the resonator. The subscripts $p$ and $r$ indicate the indices of the dofs at the connection point of the plate and resonator, respectively. Besides, the dof vector $\mathbf{u}$ is extended by adding the resonator dof as,

$$
\tilde{\mathbf{u}}=\left[\begin{array}{c}
\mathbf{u} \\
w_{r}
\end{array}\right] .
$$

Using Eq. (7a), the coupled motion between the plate and the mass-spring resonator in a unit cell can be analyzed. 
(a)

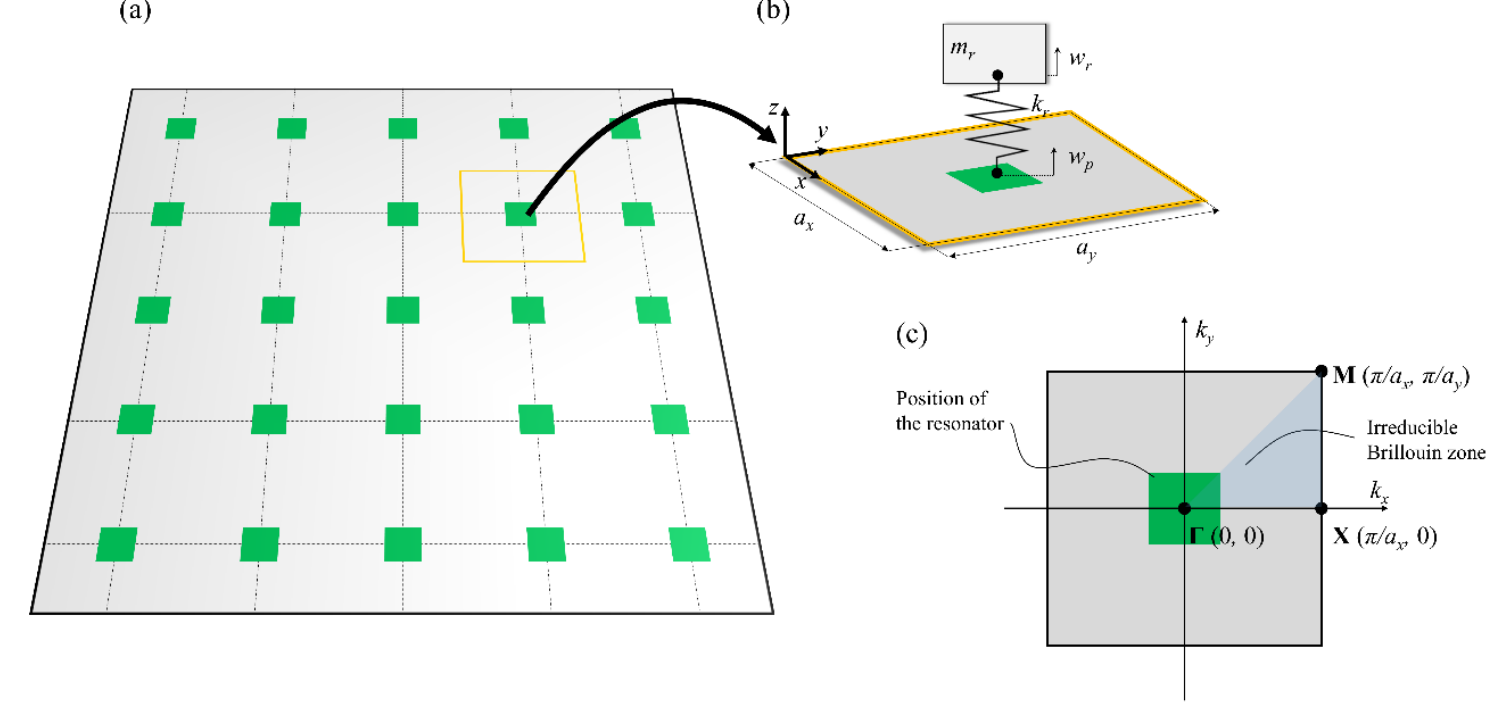

Fig. 2. The band gap structure (a), unit cell (b), and reciprocal wave vector space with the irreducible Brillouin zone (c).

With the assumption that the unit cell structure is infinitely periodic, wave functions propagating in the BG structure follow the Bloch theorem, which is stated as $[23,27]$,

$$
\mathbf{u}(\mathbf{r}+\mathbf{a})=\mathbf{u}(\mathbf{r}) \mathrm{e}^{j \mathbf{k a}}
$$

where $\mathbf{r}$ is a position vector inside a unit cell, $\mathbf{a}$ is the lattice vector $\left(\mathbf{a}=\left(a_{x}, a_{y}\right)\right)$, and $\mathbf{k}$ is the bending wave vector $\left(\mathbf{k}=\left(k_{x}, k_{y}\right)\right)$. The term $\mathrm{e}^{\mathrm{j} \mathbf{k a}}$ represents the amplitude and phase change, which is determined by the wave vector $\mathbf{k}$ and lattice vector $\mathbf{a}$.

Applying Eq. (9) on the boundaries of the unit cell, the dof vector $\mathbf{u}$ can be reduced with a reduction matrix $\mathbf{R}$ that depends on the wave vector $\mathbf{k}$ as,

$$
\begin{aligned}
& \mathbf{u}=\mathbf{R}(\mathbf{k}) \mathbf{u}^{\text {red }}, \\
& \mathbf{R}(\mathbf{k})=\left[\begin{array}{cccc}
\mathbf{e}^{\mathrm{j} k_{x} a_{x}} & 0 & 0 & 0 \\
0 & \mathbf{e}^{\mathrm{j} k_{x} a_{x}} & 0 & 0 \\
0 & 0 & \mathbf{e}^{\mathrm{j} k_{y} a_{y}} & 0 \\
0 & \mathbf{e}^{\mathrm{j} k_{y} a_{y}} & 0 & 0 \\
0 & \mathbf{e}^{\mathrm{j}\left(k_{y} a_{y}+k_{x} a_{x}\right)} & 0 & 0 \\
0 & 0 & 0 & \mathbf{I}
\end{array}\right], \\
& \mathbf{u}^{\mathrm{red}}=\left[\begin{array}{llll}
\mathbf{u}_{L} & \mathbf{u}_{L B} & \mathbf{u}_{B} & \mathbf{u}_{I}
\end{array}\right]^{T}, \\
& \mathbf{u}=\left[\begin{array}{llllll}
\mathbf{u}_{R} & \mathbf{u}_{R B} & \mathbf{u}_{T} & \mathbf{u}_{L T} & \mathbf{u}_{R T} & \mathbf{u}_{I}
\end{array}\right]^{T},
\end{aligned}
$$


where subscripts $L, R, B, T$ represent the left, right, bottom, and top boundaries, respectively, and $L B, R B, L T, R T$, I represent the left bottom, right bottom, left top, right top corners, and internal domain of the unit cell, respectively [27]. By combining Eq. (10a) with Eq. (7a), an eigenvalue equation can be obtained as follows [23],

$$
\begin{aligned}
& \mathbf{R}^{H}\left(-\omega^{2} \tilde{\mathbf{M}}+\tilde{\mathbf{K}}\right) \mathbf{R} \mathbf{u}^{\text {red }}=\mathbf{0} . \\
& \left(-\omega^{2} \mathbf{M}^{\text {red }}(\mathbf{k})+\mathbf{K}^{\text {red }}(\mathbf{k})\right) \mathbf{u}^{\text {red }}=\mathbf{0},
\end{aligned}
$$

where the superscript $H$ denotes the conjugate transpose, i.e. Hermitian operator.

Eq. (12) is the dispersion equation that states the relation between the wave vector $\mathbf{k}$ and the frequency $\omega$, i.e. the dispersion relation [23]. To obtain the dispersion relation of the BG structure, Eq. (12) needs to be solved for every possible combination of wave vectors. However, due to symmetry, we may confine the wave vectors to the irreducible Brillouin zone (IBZ), which is the triangular region in the reciprocal wave vector space as described in Fig. 2(c) [2327]. Usually, the wave vectors are further reduced to the boundary contour of the IBZ, (i.e., $\Gamma \rightarrow \mathrm{X}, \mathrm{X} \rightarrow \mathrm{M}$, and $\mathrm{M} \rightarrow \Gamma$ ) because the dispersion curve obtained by searching along the contour usually contains sufficient information to identify BGs [27]. The frequencies in the obtained dispersion curve are the frequencies at which waves propagate. On the contrary, the empty frequency bands in the dispersion curve are the BGs at which no waves propagate.

\subsection{Prediction of radiated sound pressure}

The BG structure radiates sound into the free space under a mechanical excitation as illustrated in Fig. 3(a). To estimate the radiated sound pressure, we assume the BG structure to be mounted on an infinite baffle with the exclusion of the edge diffraction of acoustic waves [34]. Under this assumption, the sound pressure $p$, which is radiated from a vibrating surface, can be calculated using the Rayleigh integral formulation as [35],

$$
p(\mathbf{r})=\frac{\mathrm{j} \omega \rho_{0}}{2 \pi} \int_{S} \frac{v_{n}\left(\mathbf{r}_{0}\right) \exp \left(-\mathrm{j} k_{\text {air }} R\right)}{R} \mathrm{~d} S,
$$

where $\rho_{0}$ is the mass density of air $\left(1.2 \mathrm{~kg} / \mathrm{m}^{3}\right), k_{\text {air }}$ is the wavenumber of acoustic wave $\left(=\omega / c_{0}\right.$, $\left.c_{0}=343 \mathrm{~m} / \mathrm{s}\right), R$ is the distance between the field point $\mathbf{r}(=(x, y, z))$ and the surface point $\mathbf{r}_{0}(=$ $(x, y, 0))$, and $v_{n}$ is the surface normal velocity. Here, the surface of the plate is considered stiff enough to neglect the air loading [36]. For many practical cases, in which the air loading is negligible, this one-way approach is valid as discussed in Ref. 34. 
(a)

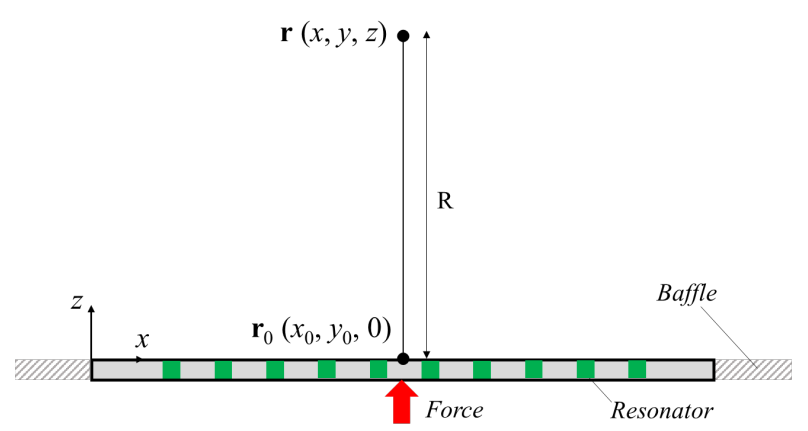

(b)

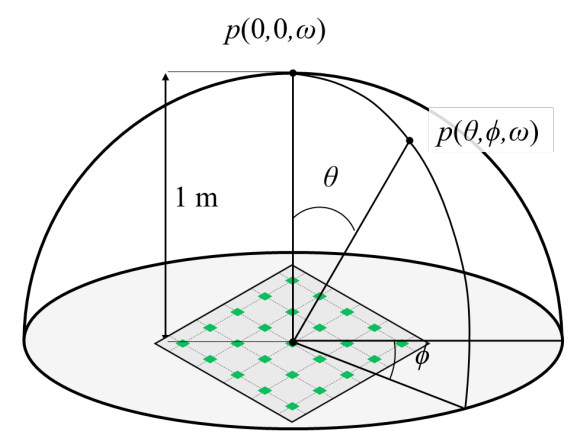

Fig. 3. Sound radiation of the baffled band gap structure (a), and the spherical coordinate for the DI calculation (b).

We employ the directivity index (DI) to evaluate the directivity of the sound radiation quantitatively as follows [37],

$$
D I(\omega)=10 \log _{10}\left(\frac{|p(0,0, \omega)|^{2}}{\frac{1}{2 \pi} \int_{\phi=0}^{2 \pi} \int_{\theta=0}^{\pi / 2}|p(\theta, \phi, \omega)|^{2} \sin \theta \mathrm{d} \theta \mathrm{d} \phi}\right),
$$

where $p(0,0, \omega)$ is the sound pressure at the point $1 \mathrm{~m}$ distant from the center of the surface, and $p(\theta, \phi, \omega)$ is the sound pressure at the point rotated by the elevation angle $\theta$ and azimuth angle $\phi$.

For the calculation of the DI, we consider a hemispherical domain since the sound field is symmetric along the $x-y$ plane due to the baffled condition [26]. Fig. 3(b) describes the coordinate system of the hemispherical domain, where the elevation angle ranges from 0 to $\pi / 2$. If the sound is radiated spatially uniform in the half space, the DI value is equal to zero, and any departure from zero DI represents the directional sound radiation [37].

\section{Numerical results}

\subsection{Test example}

We conducted numerical simulations considering a finite thin plate with periodically arranged resonators as described in Fig. 4. The geometric dimensions of the plate are $400 \mathrm{~mm}$ $\times 320 \mathrm{~mm} \times 1 \mathrm{~mm}$, and the material is aluminum with the elastic modulus $E=65 \mathrm{GPa}$, mass density $\rho=2700 \mathrm{~kg} / \mathrm{m}^{3}$, Poisson's ratio $v=0.3$, and loss factor $\eta=0.002$. All edges of the plate are clamped, and the circular surface force with the radius of $r=13 \mathrm{~mm}$ is applied. The force is placed away from the center by $50 \mathrm{~mm}$ in the $x$-axis and $25 \mathrm{~mm}$ in the $y$-axis. The aspect 
ratio of the plate and the eccentric position of the excitation are decided based on the conventional rules for the FPS design to excite as many modes as possible [38]. The resonators are arranged with a periodicity of $25 \mathrm{~mm}$, which may fill out the entire surface with $192(16 \times 12)$ square unit cells (i.e., $\boldsymbol{a}=(25 \mathrm{~mm}, 25 \mathrm{~mm}))$. The distance between the uppermost unit cell and the upper edge of the plate is $10 \mathrm{~mm}$, which is sufficiently narrow not to create edge modes within the target frequency.

The mass-spring resonator (i.e., $\mathrm{m}_{\mathrm{r}}$ and $\mathrm{k}_{\mathrm{r}}$ ) is designed to create the $\mathrm{BG}$ covering the target frequencies ranging from $300 \mathrm{~Hz}$ to $500 \mathrm{~Hz}$. Note again that other configurations of resonators can create other BG ranges and a general rule can be found in Ref. 22. For the design, we use two parameters: the resonance frequency $\left(\mathrm{f}_{\mathrm{r}}=\left(\mathrm{k}_{\mathrm{r}} / \mathrm{m}_{\mathrm{r}}\right)^{0.5} / 2 \pi\right)$, and the mass ratio $\left(\mathrm{M}_{\mathrm{r}}=\mathrm{m}_{\mathrm{r}} / \mathrm{m}_{\text {plate, }}\right.$, $\mathrm{m}_{\text {plate }}=\rho \times a_{x} \times a_{y}$ ) between the lumped mass and the plate of the unit cell. The resonance frequency $\mathrm{f}_{\mathrm{r}}$ and the mass ratio $\mathrm{M}_{\mathrm{r}}$ are chosen as $300 \mathrm{~Hz}$ and 2.375 , respectively, which open the $\mathrm{BG}$ ranging from $300 \mathrm{~Hz}$ to $549 \mathrm{~Hz}$. The two parameters determine the stiffness $\mathrm{k}_{\mathrm{r}}$ as 14.24 $\mathrm{kN} / \mathrm{m}$ and the lumped mass $\mathrm{m}_{\mathrm{r}}$ as $4 \mathrm{~g}$.

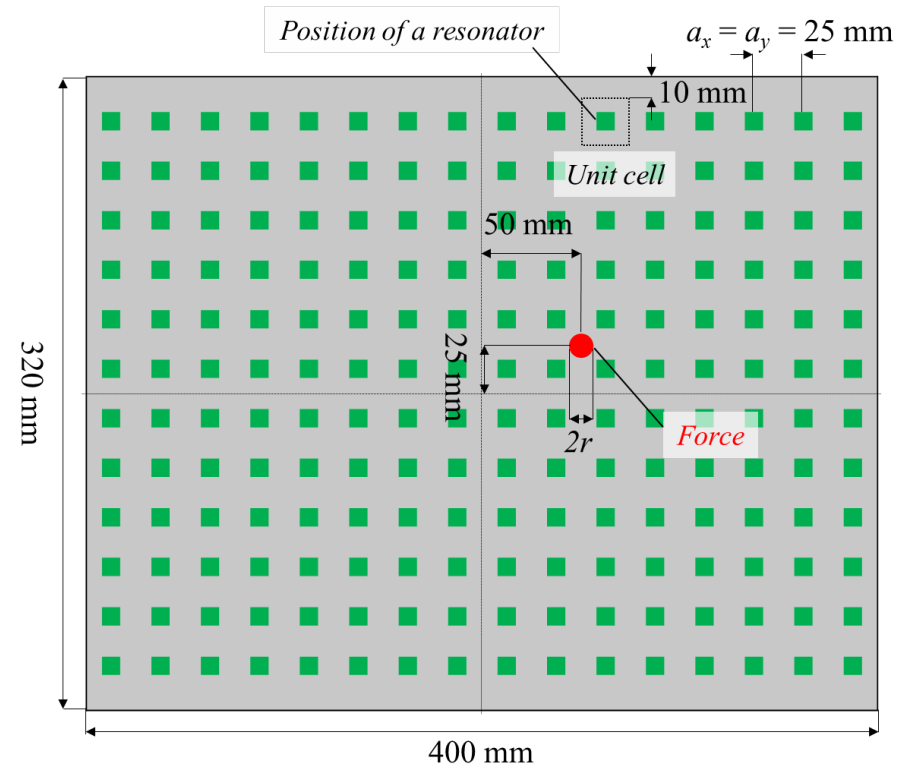

Fig. 4. Description of the target example.

\subsection{The dispersion relation and coincidence effect}

The dispersion relation of the BG structure is analyzed to investigate the interaction between the bending waves and acoustic waves. For the investigation, the dispersion curve of the bending and acoustic waves is displayed in Fig. 5(a). As shown in the figure, the group velocity of the bending wave, which is defined by the slope of the dispersion curve (i.e., $\boldsymbol{c}_{\mathrm{g}}$ $=\partial \omega / \partial \mathbf{k})$, is reduced when it asymptotes to the BG frequency due to the presence of the 
resonator [29]. The reduced velocity makes the bending wavelength coincide with the acoustic wavelength at the upper bound of the $\mathrm{BG}, 550 \mathrm{~Hz}$, which is 16 times lower than the critical coincidence frequency of a bare plate $(=9047 \mathrm{~Hz})[39]$. Focusing on the coincidence occurring at the much lower frequency than a bare plate, here, we denote this phenomenon as early coincidence.

For the further analysis of the early coincidence, the wave patterns along the $x$-axis are presented in Fig. 5(b). At point A of the dispersion curve as shown in Fig. 5(a), where the early coincidence occurs, the patterns of the bending and acoustic waves match well. At this frequency, bending wave energy transfers well to the acoustic waves due to the matched wavelengths; thus, sound radiates efficiently $[26,29]$. Therefore, using the early coincidence, the sound radiation can be amplified compared to a bare plate under the same excitation and boundary conditions. Conversely, at point B, where the bending wavenumber $(=54.2)$ is 3.4 times higher than the acoustic wavenumber $(=15.8)$, the wave patterns mismatch considerably. Here, the point $\mathrm{B}$ is chosen for the clear comparison purpose emphasizing the mismatch between acoustic and bending wavelengths. For this case, the enhancement of sound radiation from the efficient interaction between bending and acoustic waves is unattainable. 
(a)

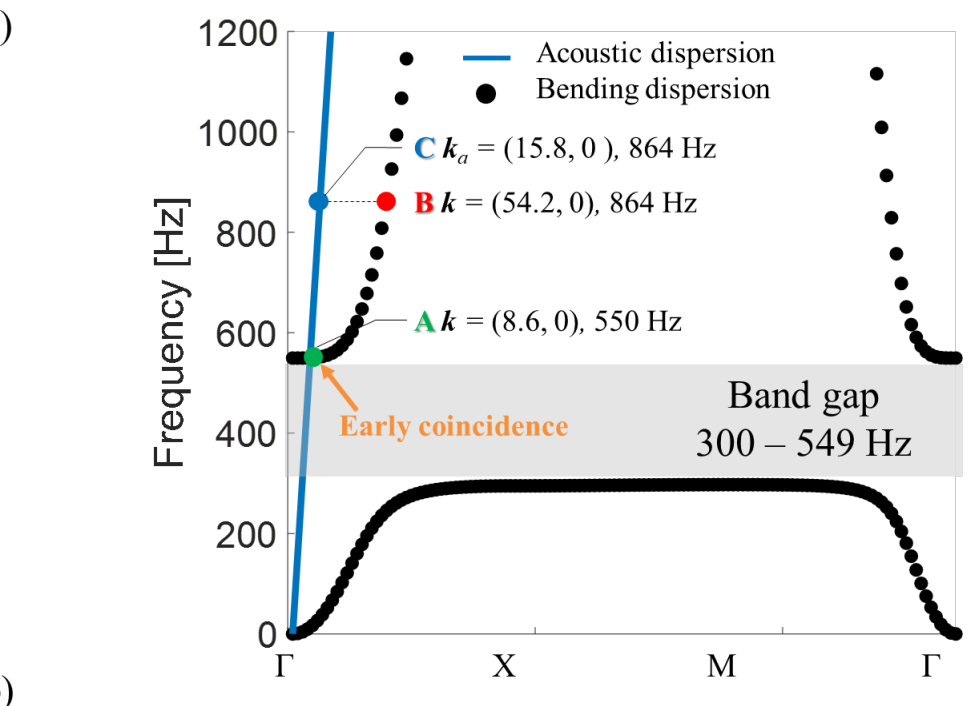

(b)
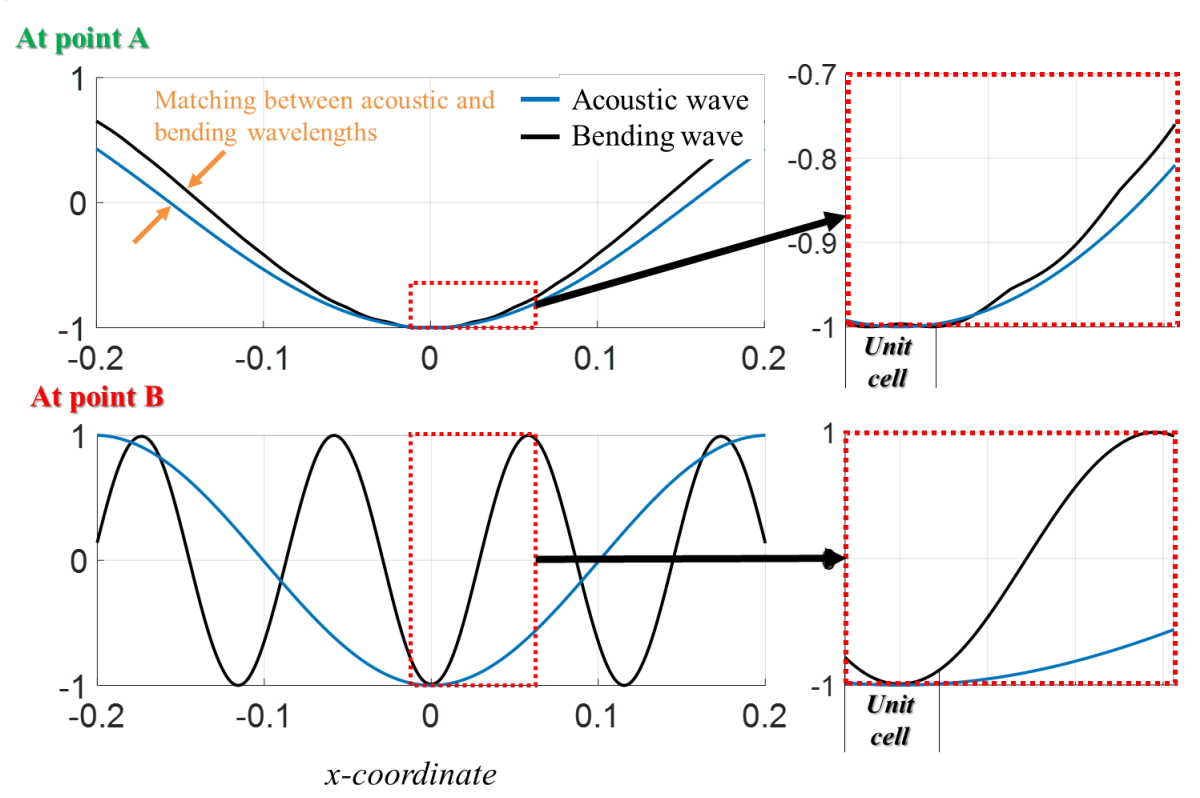

Fig. 5. Dispersion curves of the bending wave and acoustic wave (a), and wave patterns along the $x$-coordinate (b).

\subsection{Mode elimination using the band gap}

Here, we investigate how the BG structure eliminates the modes. For this investigation, the eigenfrequency spectra of the bare plate and the BG structure are analyzed. Fig. 6 displays the eigenfrequencies of the BG structures with three different boundary conditions (i.e., free, clamped, and simply-supported) together with the bare plate. In contrast to the bare plate, which has distributed eigenfrequencies throughout the entire frequencies, the BG structures have no eigenfrequencies in the $\mathrm{BG}$ range, $300 \mathrm{~Hz}-549 \mathrm{~Hz}$. Here, we denote this frequency band as a mode-free band. The mode-free band appears regardless of the boundary conditions since the 
BG attenuate the bending wave exponentially before it reaches the boundaries. Therefore, the mode-free band can be considered as a property of BG structures, which controls resonances and vibration shapes. This attractive feature of the mode-free band has received attention in previous studies [23-29]. However, in this paper, we exploit the mode-free band to reduce peaks and achieve a spectrally smooth sound radiation, a feature which we believe has not been considered previously.

(a)

(b)

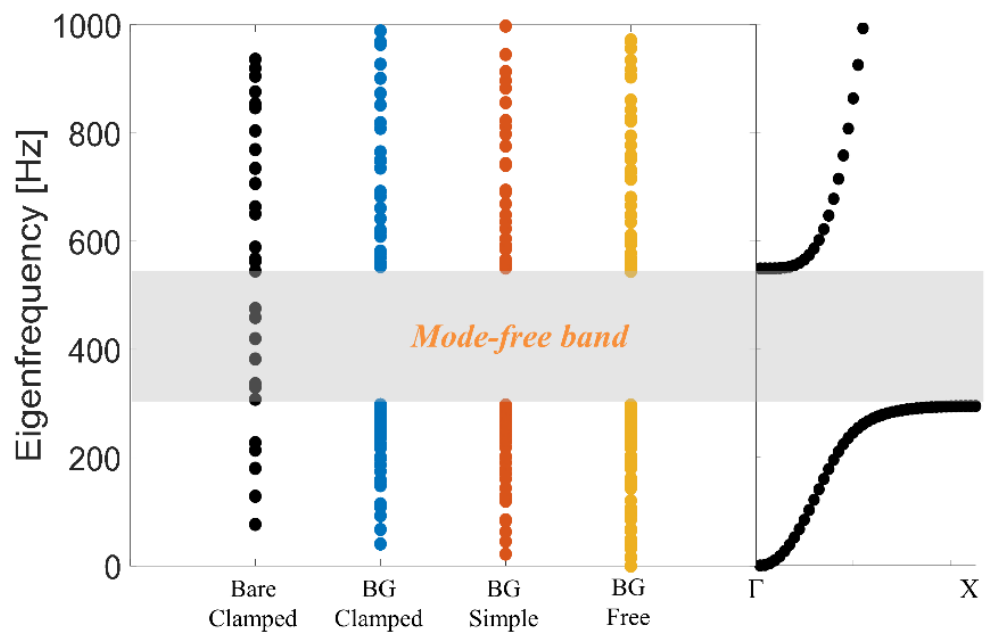

Fig. 6. Eigenfrequency spectra of the band gap structures (a) and the dispersion curve (b).

\subsection{Vibro-acoustic responses of the band gap structure}

The sound radiation of the BG structure is investigated to analyze the effect of the modefree band on the sound radiation. For this purpose, sound pressure levels (SPLs) at a point $1 \mathrm{~m}$ distant from the center of the BG structure (i.e., On-axis) are calculated using Eq. (13). The BG structure is excited by a surface force of $1 \mathrm{~N} / \mathrm{m}^{2}$, and for the damping of the resonator, an isotropic loss factor of 0.02 is used.

Fig. 7 displays the radiated SPLs of the bare plate and BG structure. In this figure, three features of the BG structure distinguish significantly from the bare plate. First, the SPL of the BG structure is smooth within the BG range, which corresponds to the mode-free band. On the contrary, the SPL of the bare plate fluctuates considerably due to well separated modes. Second, at the upper bound of the BG, the SPL peak appears. The peak appears at the early coincidence frequency. Third, at the lower bound of the BG, a dip appears. The dip occurs due to the significant reduction of vibrations, and usually, this feature has been exploited for noise attenuation $[24,25,27]$. However, for loudspeaker applications, the SPL needs to be amplified to achieve a flat spectrum. 


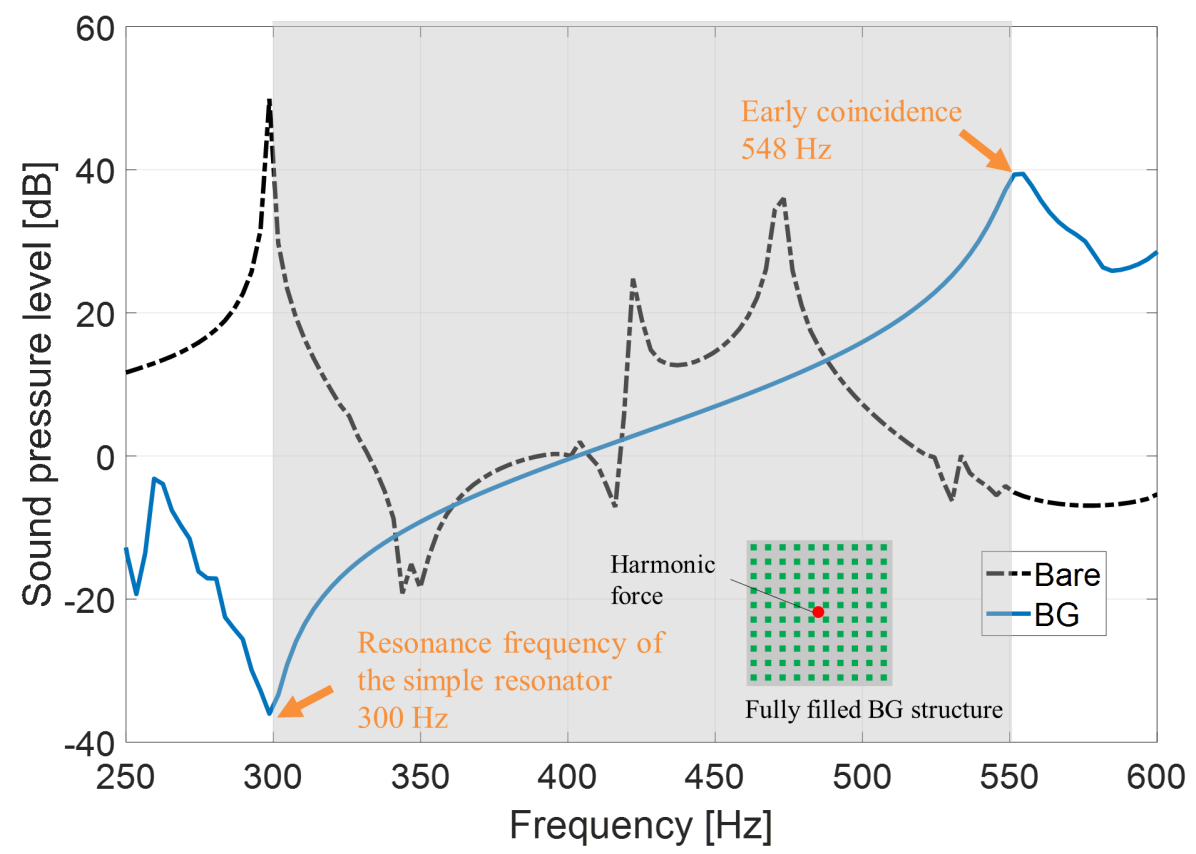

Fig. 7. On-axis sound pressure level at $1 \mathrm{~m}$ distant from the band gap structure.

To amplify the SPL at the lower bound of the BG, we use a strategy that creates a localized mode within the BG close to the dip frequency. The creation of the localized mode relies on confining vibrations around the excitation force by making a square sound radiation zone where resonators are removed [26]. The effect of the zone size is investigated by analyzing the eigenmodes of the BG structure with three different edge lengths of the zone being $50 \mathrm{~mm}, 100$ $\mathrm{mm}$, and $125 \mathrm{~mm}$.

Fig. 8 displays the localized modes obtained from the eigenvalue analysis of the BG structure. The smallest zone with the edge length of $50 \mathrm{~mm}$ has a localized mode at $489 \mathrm{~Hz}$ with the piston-like mode shape, i.e. $(1,1)$ mode. On the other hand, for the biggest zone with the edge length of $125 \mathrm{~mm}$, the lowest localized mode appears at $305 \mathrm{~Hz}$, which is close to the SPL dip. Moreover, higher order modes emerge at $437 \mathrm{~Hz}$ and $537 \mathrm{~Hz}$ with more complicated mode shapes. Using these localized modes, the amplification of the SPL dip is investigated.

Fig. 9 displays the calculated SPLs of the BG structure with the designated sound radiation zone. As shown in the figure, the small zone with the edge length of $50 \mathrm{~mm}$ produces little amplified SPL due to the localized mode at $489 \mathrm{~Hz}$, but a similar dip appears at the lower bound of the BG. On the other hand, for the larger zones with edge lengths of $100 \mathrm{~mm}$ and $125 \mathrm{~mm}$, the dip disappears owing to the peaks associated with the localized modes at $347 \mathrm{~Hz}$ and 305 $\mathrm{Hz}$ that are close to the dip frequency. Furthermore, for these cases, SPLs are amplified 
considerably over the bare plate with the peaks in the BG range. Despite the peaks within the $\mathrm{BG}$, the separated location of peaks still improves the flatness of the frequency response significantly in comparison with the bare plate.

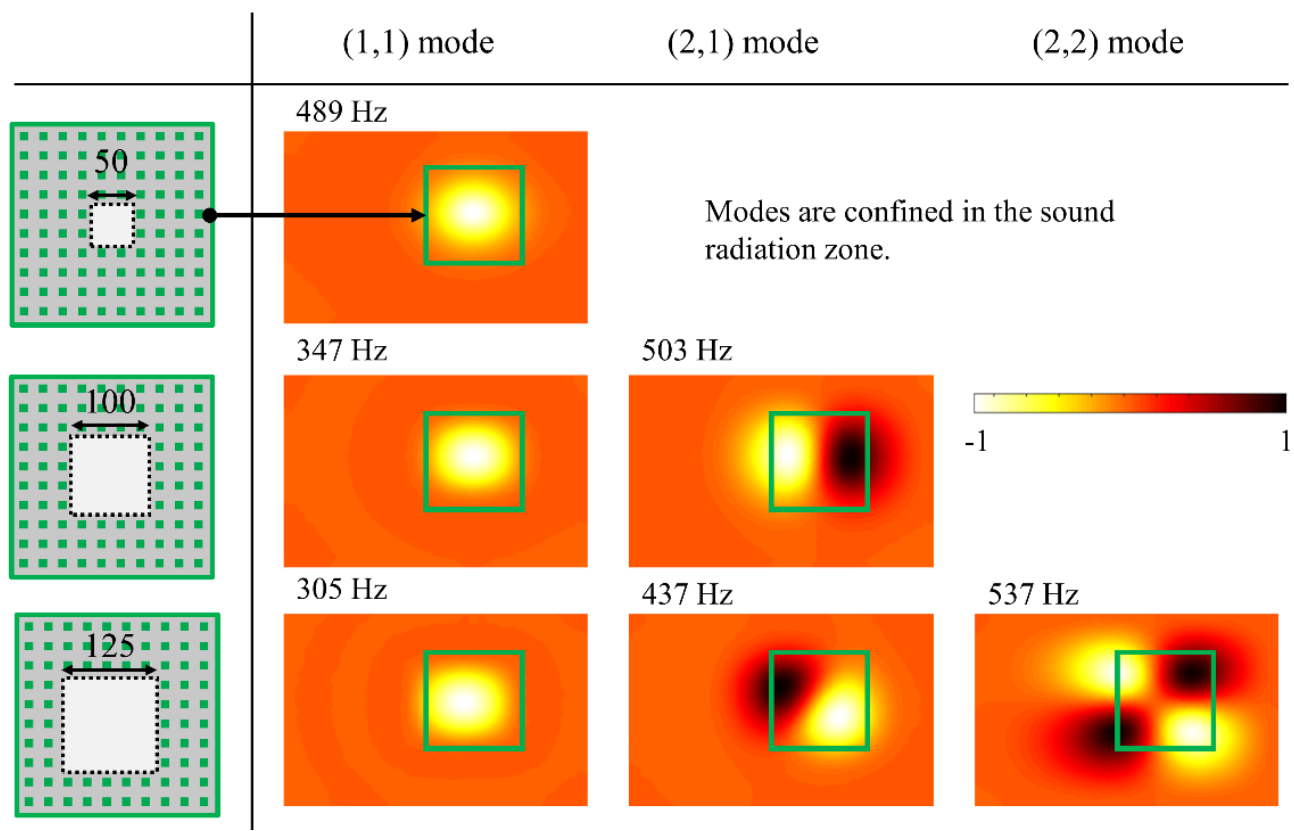

Fig. 8. Localized modes formed inside of the sound radiation zone of the band gap structure where the green squares represent the adjacent regions of the sound radiation zone.

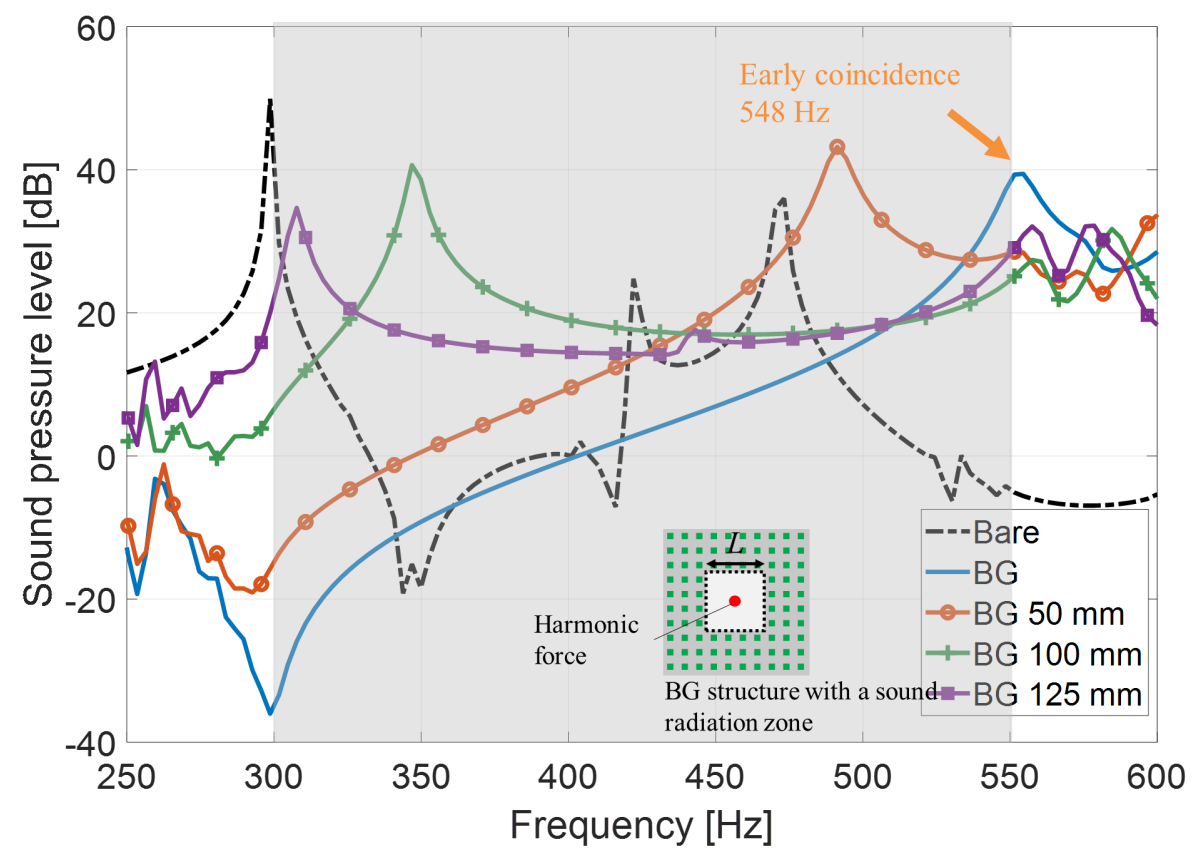

Fig. 9. Sound pressure level of the band gap structure with a square sound radiation zone. 
One further benefit of the BG structure is a spatially uniform sound radiation, which is hard to achieve in ordinary structures due to complex mode shapes [26]. For the evaluation of the directivity of sound radiation, the DIs are calculated and displayed in Fig. 10(a). As shown in the figure, the bare plate has a strongly fluctuating DI, which illustrates the highly directional sound radiation. Conversely, the BG structures have smooth DIs close to zero within the BG, indicating the spatially uniform sound radiations in the hemispherical space. However, one dip of the DI appears at $440 \mathrm{~Hz}$ for the $125 \mathrm{~mm}$ sound radiation zone. This frequency corresponds to the $(2,1)$ localized mode, in which the half-plane vibrates out of phase, as shown in Fig. 8. However, the $100 \mathrm{~mm}$ sound radiation zone has no such dip because the $(2,1)$ mode is located close to the upper bound of the BG where the early coincidence occurs.

To supplement the DI calculation in Fig. 10(a), the cross-sectional sound fields on the $x-z$ plane are visualized. Fig. 10(b) illustrates the sound fields of the $100 \mathrm{~mm}$ sound radiation zone at three frequencies $320 \mathrm{~Hz}, 500 \mathrm{~Hz}$, and $542 \mathrm{~Hz}$, at which significant deviations of the DIs appear for the bare plate. As can be seen in the figure, the BG structure shows the uniform sound radiation at three frequencies, whereas the bare plate has the highly directional sound radiation. 
(a)

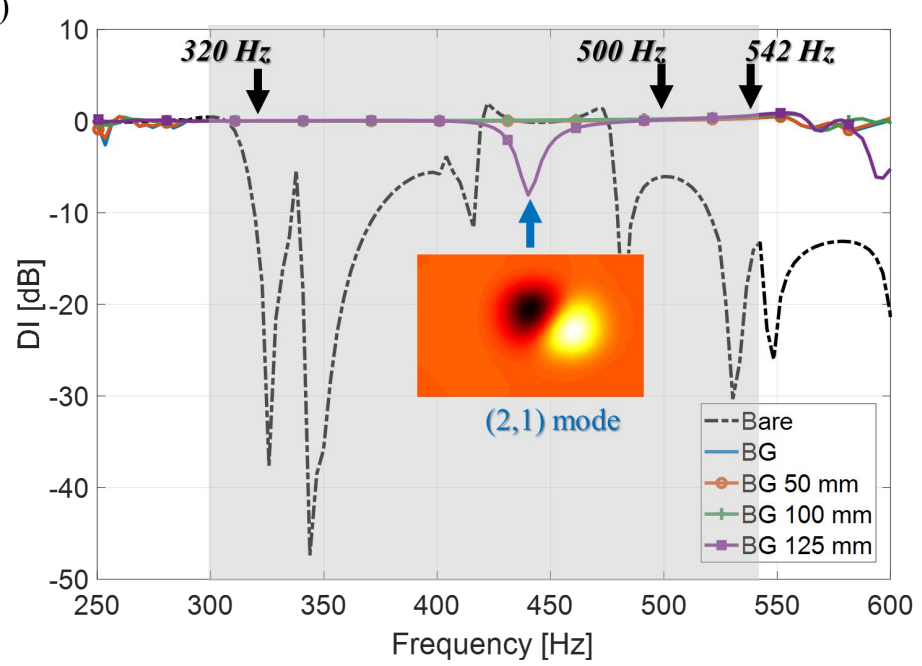

(b)
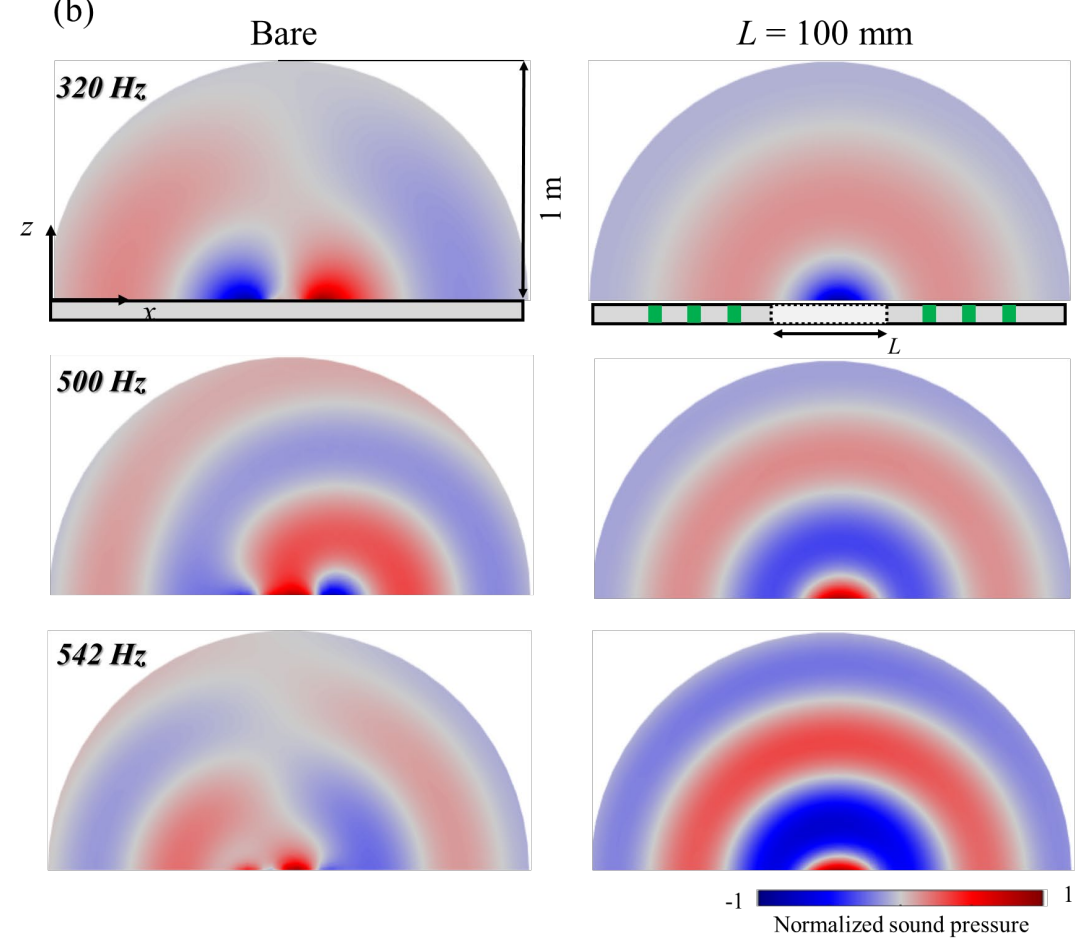

Fig. 10. Directivity index for five cases (a), and sound fields radiated from the bare and the band gap structure (b).

\subsection{The required number of resonators for the band gap realization}

The number of resonators that constitutes the BG structure should be sufficient to create the BG within the frequency range of interest. For the realization of the BG structure with fully filled resonators, 192 resonators are used. However, this considerable number of resonators is impractical and not necessarily required to take advantage of the BG [24,27].

For the determination of the sufficient number of resonators to realize the BG structure, the SPLs are calculated with a different number of resonators. Fig. 11 displays the SPL results of 
the BG structure with a $100 \mathrm{~mm}$ sound radiation zone. In the figure, $\mathrm{n}$ denotes the depth of the resonator arrangement that bounds the sound radiation zone, and six values of $n(=1,2,3,4$, 5 , and 8 which is the maximum) are considered. It can be seen in the figure that as $n$ increases, the SPLs converge to the case of the maximum $n(=8)$. However, for $n>3$, the SPLs rarely vary within the target frequencies ranging from $300 \mathrm{~Hz}$ to $500 \mathrm{~Hz}$. Hence, for this specific case, $84(=10 \times 10-4 \times 4)$ resonators, which correspond to the case of $n=3$ is seen to be sufficient to achieve the benefits of the BG structure.

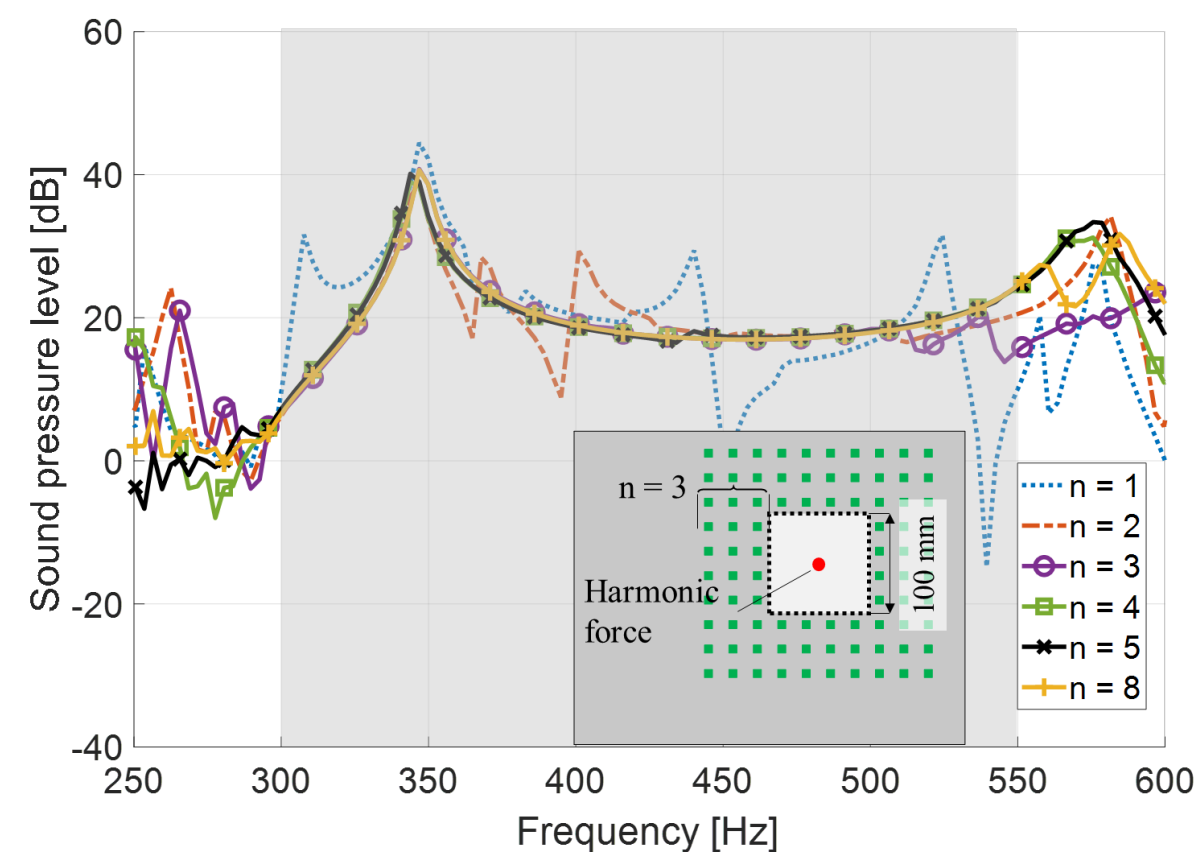

Fig. 11. Effect of number of resonators on the sound pressure level.

\section{Experimental results}

\subsection{Resonator design and fabrication}

To experimentally validate the numerical results, the BG structure is made with a cantilever resonator illustrated in Fig. 12(a). The cantilever resonator is made of acrylic material with the elastic modulus $E=3.1 \mathrm{GPa}$, mass density $\rho=1165 \mathrm{~kg} / \mathrm{m}^{3}$, Poisson's ratio $v=0.38$. The tip mass of the resonator weighs $5.2 \mathrm{~g}$, and is assumed rigid in the frequency range of interest. The dimensions of the resonator are determined to obtain the BG ranging from $308 \mathrm{~Hz}$ to $471 \mathrm{~Hz}$, which is close to the target frequencies. Fig. 12(b) displays the dispersion curve and the BG of the unit cell, which is obtained by a three-dimensional (3D) FE simulation using COMSOL Multiphysics. Fig. 12(c) and (d) display the Bloch mode shapes at the lower and upper bounds 
of the BG. The Bloch mode shapes where the cantilever resonances are dominant, demonstrate that the cantilever resonator opens the BG when it is arranged periodically.

(a)

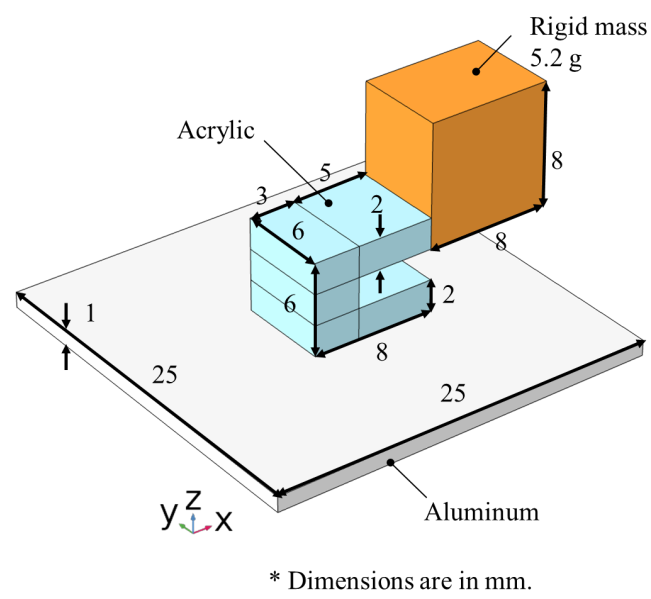

(c) Lower bound $308 \mathrm{~Hz}$

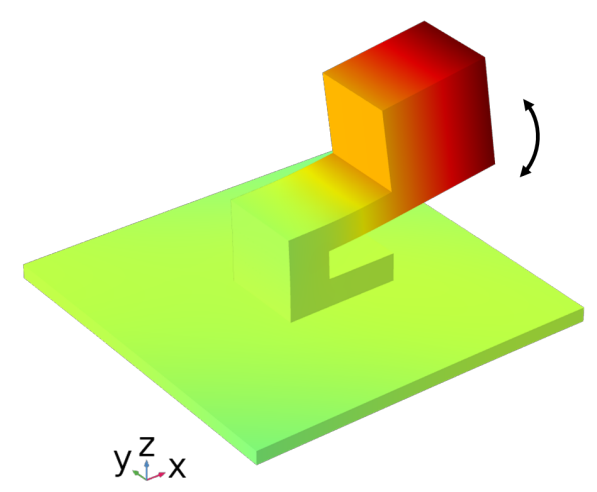

(b)

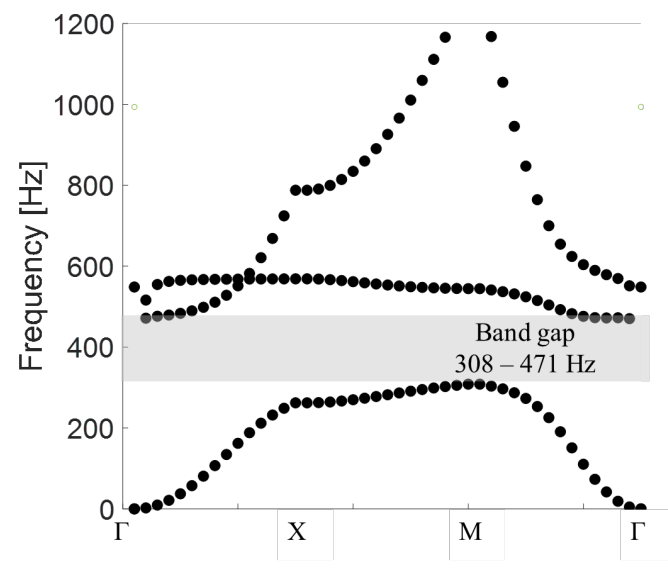

(d) Upper bound $471 \mathrm{~Hz}$

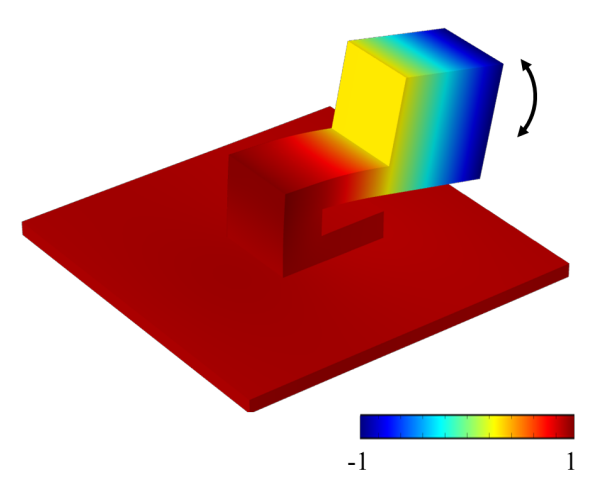

Fig. 12. The unit cell with the cantilever resonator (a), dispersion curve (b), and Bloch mode shapes at the lower bound (c) and upper bound (d) of the band gap.

The cantilever resonators are fabricated using laser cutting of a transparent acrylic sheet of $6 \mathrm{~mm}$ thickness, and the tip mass is made of a pair of a bolt and a nut, which weighs $5.2 \mathrm{~g}$. The cantilever resonators are arranged on a $1 \mathrm{~mm}$ thick aluminum plate with a periodicity of 25 $\mathrm{mm}$, as displayed in Fig. 13. A square sound radiation zone with $100 \mathrm{~mm}$ edge length is made, and it is bounded by 84 resonators that are sufficient to create the BG. For the attachment of the resonator on the aluminum plate, silicon glue is used. An electrodynamic exciter (Dayton Audio Daex 13CT) is attached to the center of the sound radiation zone. All edges of the aluminum plate are clamped using the $30 \mathrm{~mm}$ thick wooden frame.

For the evaluation of the deviation between the FE model and fabricated device, the resonance frequencies of cantilever resonators are measured for five different samples. With 
the fixed boundary condition on the floor of the resonator, the 3D FE simulation results in the first and second resonance frequencies as $332 \mathrm{~Hz}$ and $587 \mathrm{~Hz}$, respectively. These two resonance frequencies are compared with the measurement results in Table 1. As shown in the table, the measurement results agree well with the simulation with deviations (Dev.) smaller than $4 \%$.

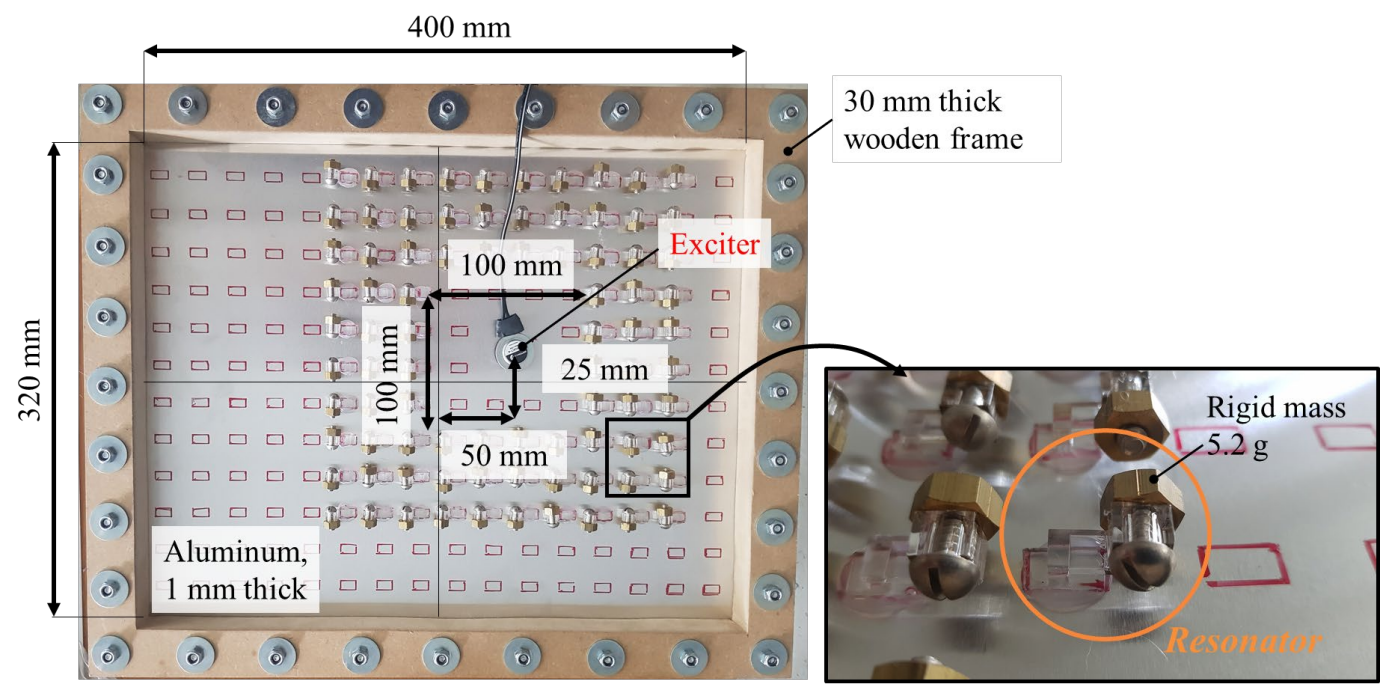

Fig. 13. Fabricated band gap structure and cantilever resonator.

Table 1. Resonance frequencies measured for five different resonator samples.

\begin{tabular}{lll}
\hline Sample \# & $\mathbf{1}^{\text {st }}$ resonance $[\mathbf{H z}]$ & $\mathbf{2}^{\text {nd }}$ resonance $[\mathbf{H z}]$ \\
\hline 1 & 323 & 560 \\
2 & 355 & 571 \\
3 & 334 & 565 \\
4 & 325 & 553 \\
5 & 349 & 584 \\
\hline Avg. (Dev.) & $337(1.5 \%)$ & $566(3.6 \%)$ \\
\hline
\end{tabular}

\subsection{Vibration measurement}

Vibration measurements are carried out for analysis of the vibrational response of the fabricated BG structure. The vibrational velocity distribution on the BG structure is measured using the laser Doppler vibrometer (Polytec PSV-400) with pseudo-random signal ranging from $10 \mathrm{~Hz}$ to $10 \mathrm{kHz}$. The scanning resolution is $11 \mathrm{~mm}$ (i.e., $35 \times 33=1155$ points), which is dense enough to capture the bending wavelength of $35 \mathrm{~mm}$ at $10 \mathrm{kHz}$. Fig. 14(a) describes the vibration measurement setup and Fig. 14(b) and (c) display the measured root mean squared (RMS) velocity fields averaged over the frequency ranging from $10 \mathrm{~Hz}$ to $10 \mathrm{kHz}$. In contrast 
to the distributed vibrations on the entire bare plate, the BG structure displays the desired localized vibration pattern around the sound radiation zone.

Fig. 15(a) displays the velocity transfer functions (Hs) averaged over all measurement points. At the frequencies lower than the BG, the two responses are similar in the way that the averaged vibrations fluctuate with many resonances. On the other hand, in the BG range, the velocity of the BG structure decreases and become smooth. The significantly reduced vibration level within the BG can be considered as evidence of the BG existence $[23,24,27,28]$.

Moreover, two clear peaks appear for the BG structure at 337 and $428 \mathrm{~Hz}$. These two peaks correspond to the localized modes created in the sound radiation zone as predicted in the numerical simulations in Fig. 8. Fig. 15(b) presents the vibration patterns at six different frequencies, including the localized modes: 250, 284, 337, 398, 428, and $489 \mathrm{~Hz}$. The figure shows the change of the vibration patterns for different frequencies and the vibration localization within the $\mathrm{BG}$ range.

(a)

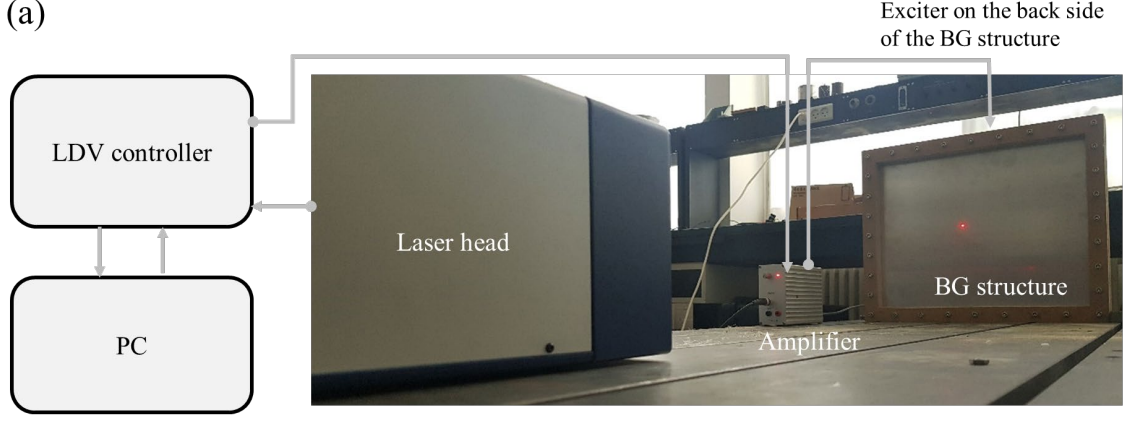

(b) Bare

(c) Band gap
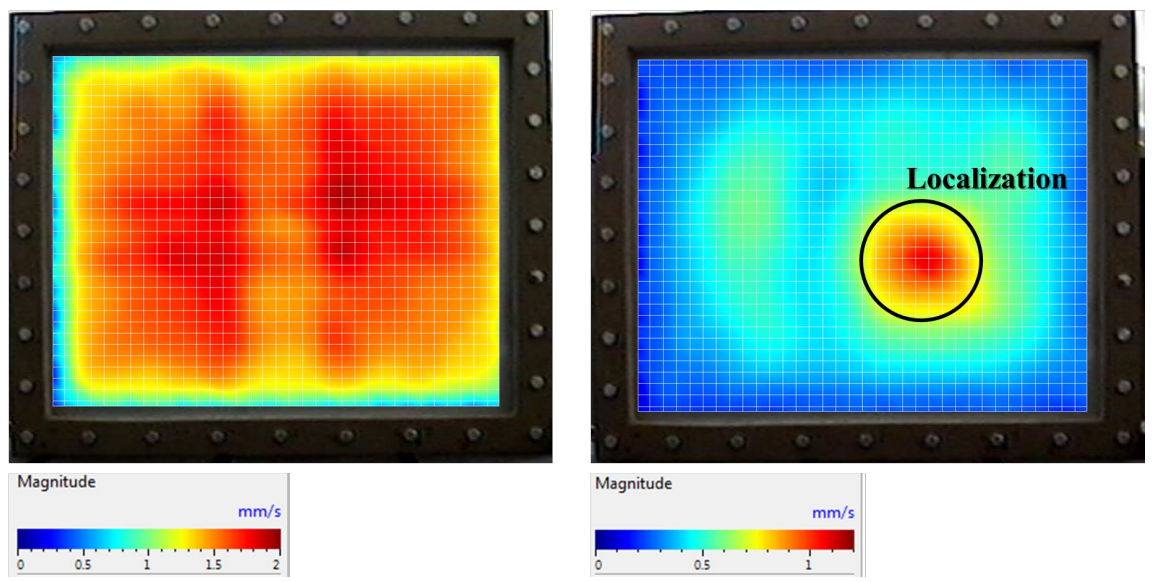

Fig. 14. Measurement setup (a) and RMS normal velocity fields of the bare plate (b), and band gap structure (c). 
(a)

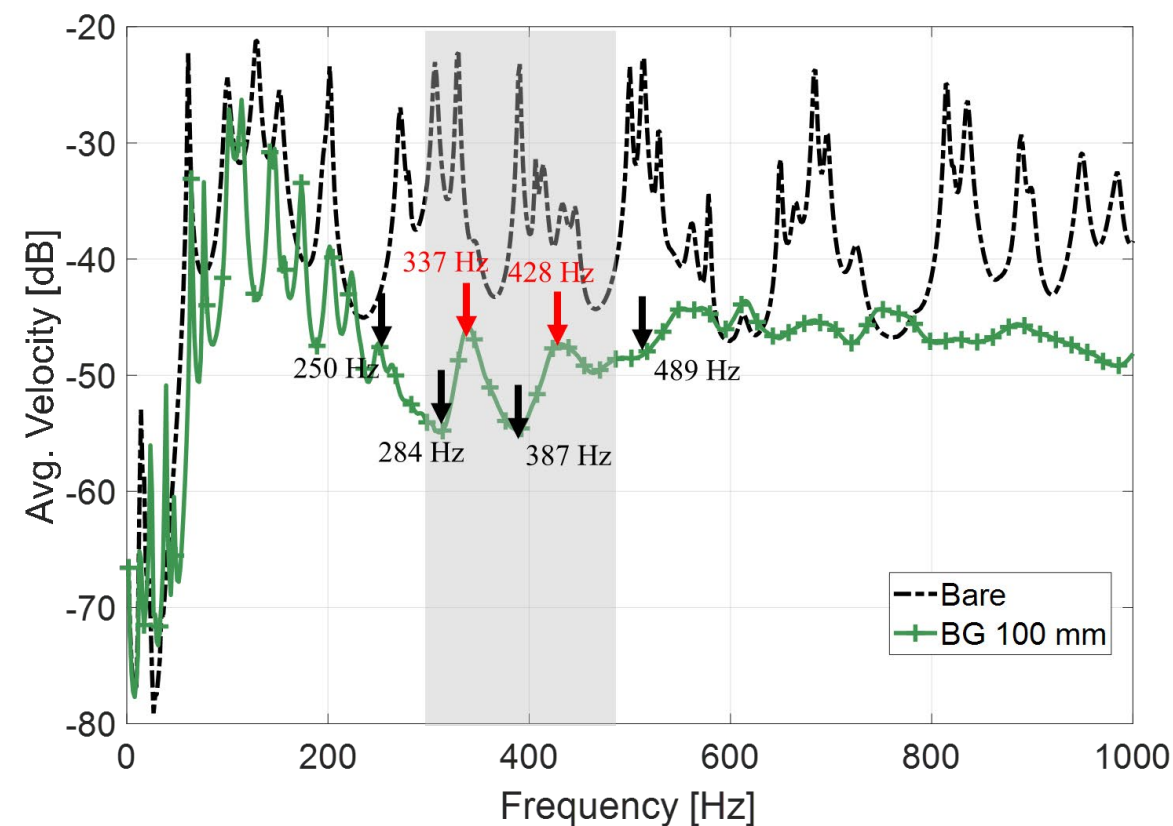

(b)
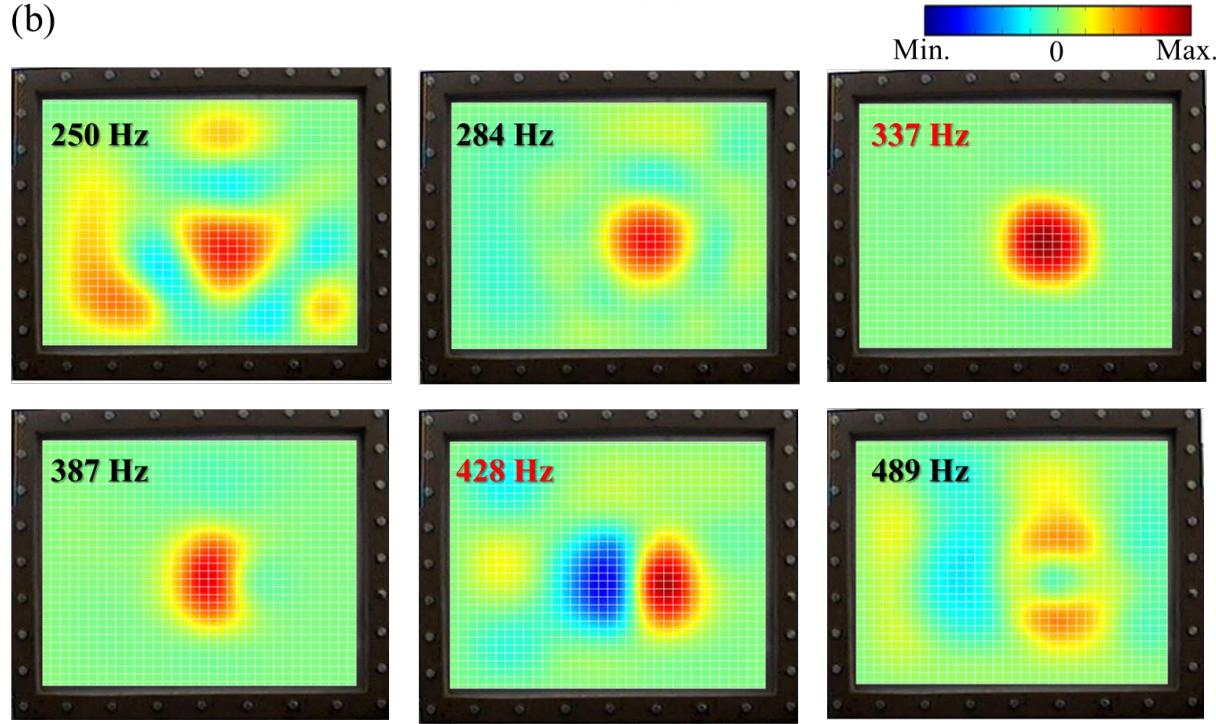

Fig. 15. Averaged velocity transfer function $\mathrm{Hs}(\mathrm{a})$, and the vibration pattern within the band gap ranges (b).

\subsection{Acoustic measurement}

An acoustic measurement is carried out in an anechoic chamber $\left(60 \mathrm{~m}^{3}\right.$, cutoff frequency of $100 \mathrm{~Hz}$ ) to validate the radiated SPLs. A microphone (B\&K Type 4192) is located at $1 \mathrm{~m}$ distance from the center of the BG structure. The BG structure is mounted on a base, which can rotate with an interval of 15 degrees in the azimuth angle $\theta$. The input signal that excites the structure is a multi-tone ranging from $20 \mathrm{~Hz}$ to $20 \mathrm{kHz}$. Using the input electrical signal, v, 
as the reference, the acoustic transfer function $\mathrm{H}_{\mathrm{pv}}$ is calculated. Fig. 16 describes this acoustic measurement setup.

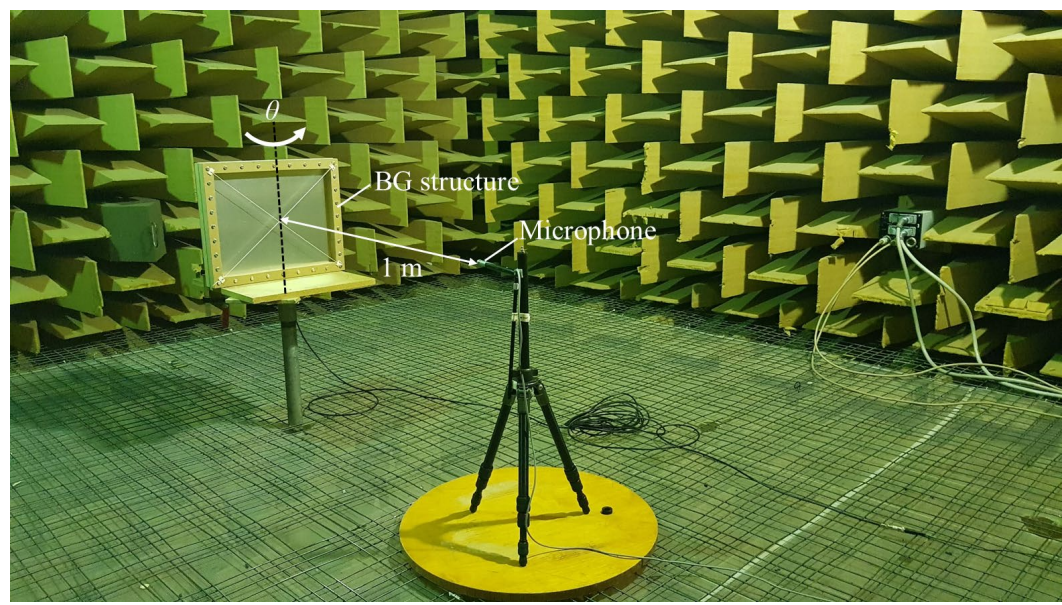

Fig. 16. Acoustic measurement setup in the anechoic chamber.

Fig. 17 displays the SPL of the bare plate and BG structure. The bare plate shows strongly fluctuating responses with many peaks and dips in the entire frequency range of interest. On the other hand, the BG structure shows much more smoothed responses because the modes are eliminated within the BG range. One peak appears at $337 \mathrm{~Hz}$, which corresponds to the localized mode, as can be seen in Fig. 15. This peak amplifies the sound pressure by $14 \mathrm{~dB}$ at the frequencies close to the lower bound of the BG: 300 - $350 \mathrm{~Hz}$. However, the effect of the other localized mode at $428 \mathrm{~Hz}$ is negligible since the exciter is placed near the nodal line.

For the quantitative evaluation of the amplification and smoothing of the BG structure, the overall and standard deviation of the SPL are compared in Table 2. As shown in the table, the BG structure amplifies the overall SPL by $5.5 \mathrm{~dB}$ with the standard deviation reduced by 5.7 $\mathrm{dB}$, which shows the benefits of the BG structure for the sound radiation purpose. 


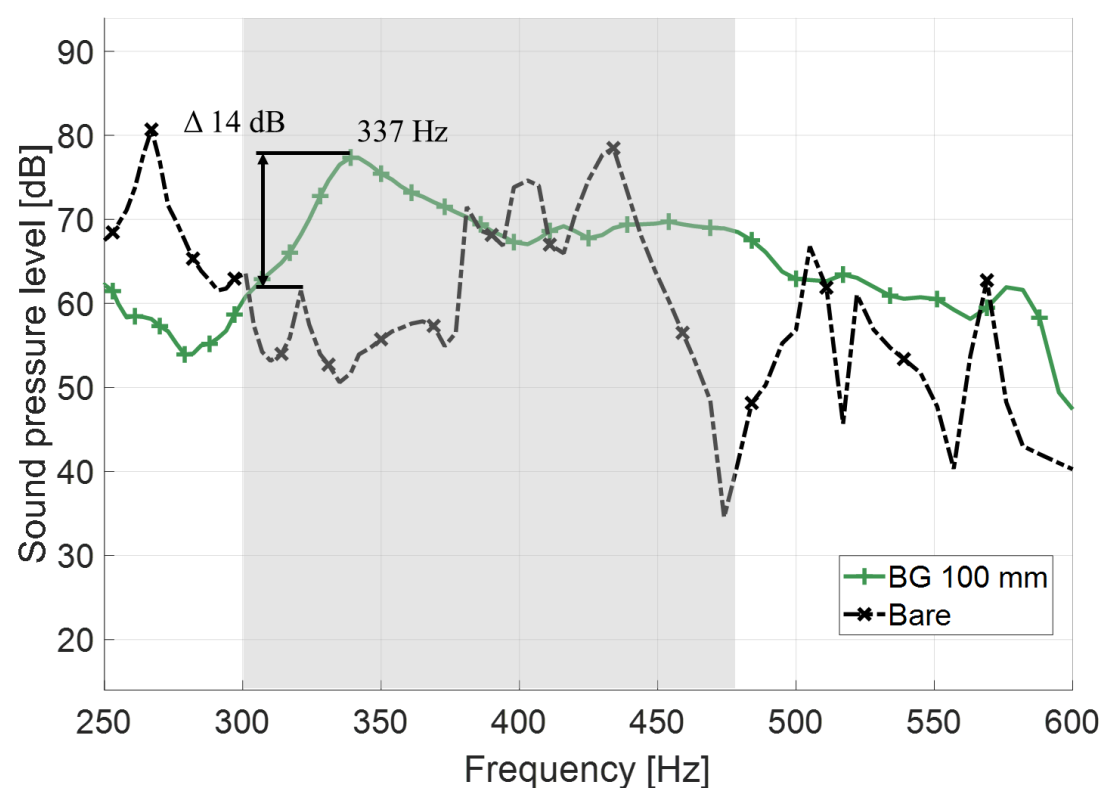

Fig. 17. Acoustic response at the point $1 \mathrm{~m}$ distant from the center of the structures.

Table 2. Comparison of SPL between the bare and the band gap structure in the target frequency range $(300 \mathrm{~Hz}-500 \mathrm{~Hz})$.

\begin{tabular}{lll}
\hline & Overall SPL $[\mathrm{dB}]$ & Standard deviation $[\mathrm{dB}]$ \\
\hline Bare & 81.6 & 9.6 \\
BG & 87.1 & 3.9 \\
\hline Improvement & 5.5 & 5.7 \\
\hline
\end{tabular}

To analyze the directivity of the sound radiation, SPLs are measured at different azimuth angle. For the measurement, the azimuth angle variates from 0 degrees to 60 degrees with an increment of 15 degrees in order to capture directivity of the sound radiation. Fig. 18(a) and (b) display the measurement results of the bare plate and BG structure, respectively. The SPL from the bare plate varies up to $12 \mathrm{~dB}$ for different azimuth angles, which indicates the directional sound radiation. On the other hand, the BG structure has small variations of the SPL up to $2 \mathrm{~dB}$ from 0 degrees to 45 degrees, supporting the numerical predictions of improved sound radiation directivity, as shown in Fig. 10. The variation increases to $6 \mathrm{~dB}$ at 60 degrees, which may indicate a leakage of sound to the back of the plate due to the absence of an infinite baffle. 
(a)

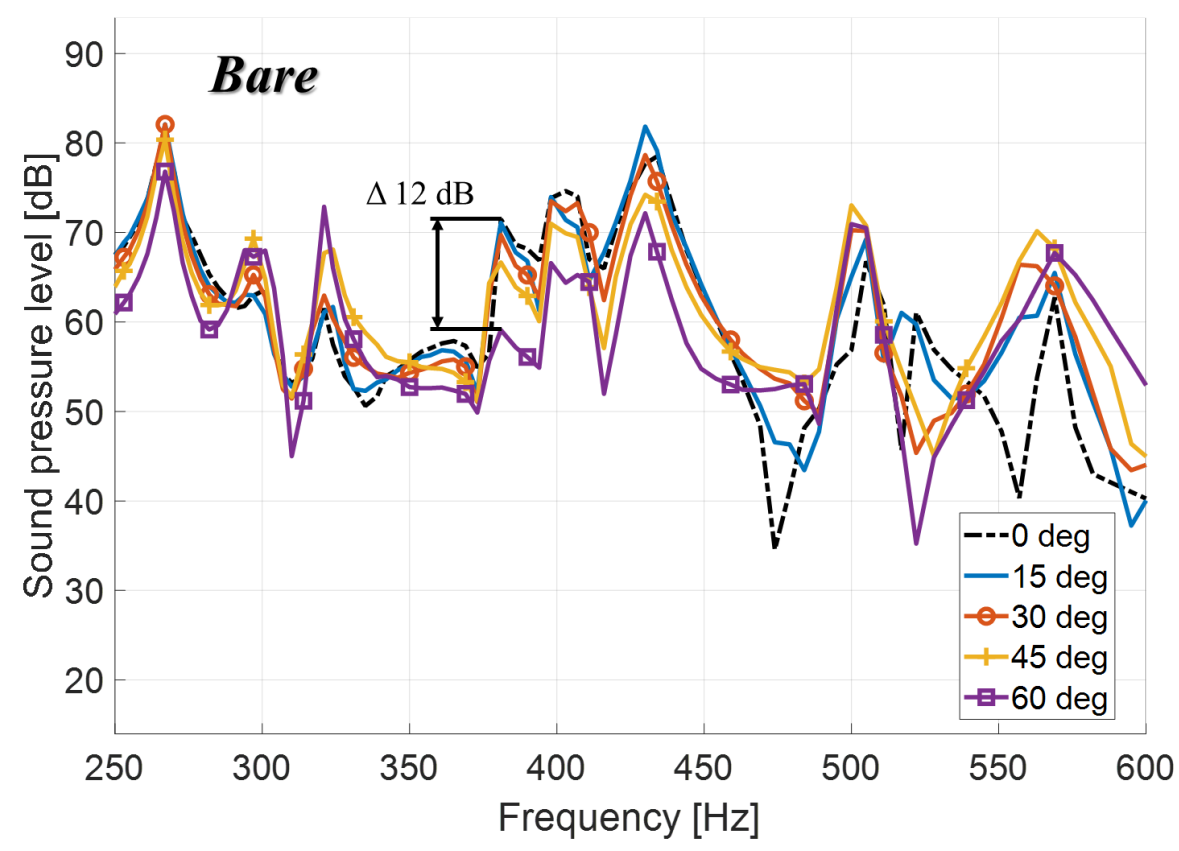

(b)

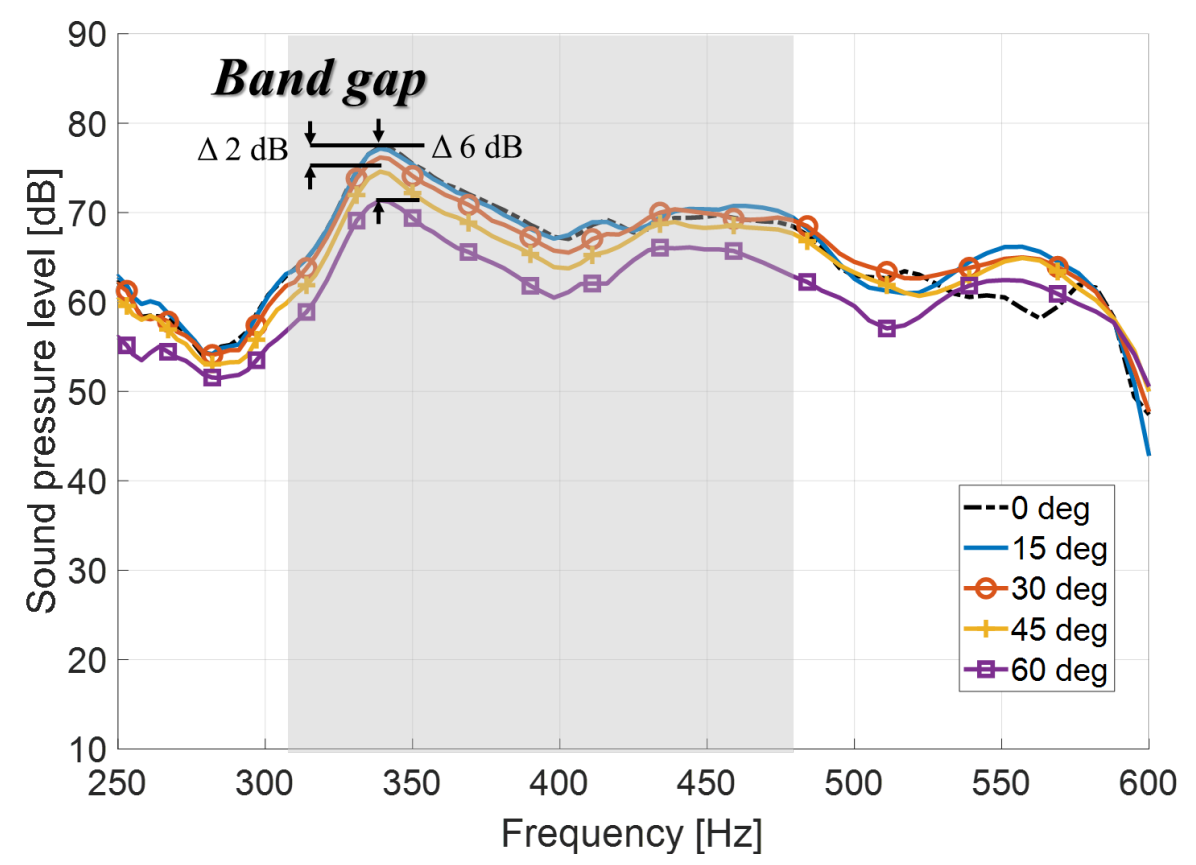

Fig. 18. Acoustic response of the bare (a) and band gap structure (b) at the points $1 \mathrm{~m}$ distant from the center of the structures with azimuth angle $\theta$.

\section{Conclusion}

We present a novel technique, which eliminates modes of a thin plate structure using the BG in order to achieve a smooth frequency spectrum and spatially uniform sound radiation. Using numerical simulations, we verify that the BG structure has a mode-free band, resulting 
in a smooth sound radiation. To amplify the radiated sound pressure, a square sound radiation zone without resonators, is created. The modified BG structure amplifies the sound pressure considerably while maintaining the smoothness in the designated frequency spectrum. Moreover, the BG structure shows a significantly improved directivity with DI values close to zero, demonstrating a spatially uniform sound radiation in the hemispherical space, which is difficult to achieve in a bare plate.

An experimental validation of the acoustic radiation of the BG structure confirms that the BG structure amplified the overall SPL by $5.5 \mathrm{~dB}$ and reduced the standard deviation by 5.7 $\mathrm{dB}$ in the frequency range from $300 \mathrm{~Hz}$ to $500 \mathrm{~Hz}$. Furthermore, the BG structure shows smaller variations of the sound pressure for different azimuth angles. The suggested mode elimination technique using a BG structure can improve the sound radiation with more uniform and effective sound radiation in a designed frequency range.

\section{Acknowledgement}

This work was supported by the Technical University of Denmark (Signature project).

\section{Reference}

[1] H. B. Juul-Nyholm, J. C. Severinsen, H. Schneider, N. H. Mortensen, and M. A. E. Andersen, Construction of Lightweight Loudspeaker Enclosures, in: Proceedings of the $142^{\text {nd }}$ Audio Engineering Society Convention, 2017, No. 9784.

[2] M. Tashiro, G. Bank, and M. Roberts, A new flat panel loudspeaker for portable multimedia, in: Proceedings of the $103^{\text {rd }}$ Convention of Audio Engineering Society, 1997, No. 4527.

[3] J.-H. Woo and J.-G. Ih, Generation of a Virtual Speaker and Baffle on a Thin Plate Controlled by an Actuator Array at the Boundary, IEEE-ASME T. Mech. 24 (3) (2019) 1197-1207.

[4] N. Harris, and M. J. Hawksford, The Distributed-Mode Loudspeaker (DML) as a Broadband Acoustic Radiator, in: Proceedings of the $103^{\text {rd }}$ Convention of Audio Engineering Society, 1997, No. 4526.

[5] M. R. Bai, and T. Huang, Development of panel loudspeaker system: Design, evaluation and enhancement, J. Acoust. Soc. Am. 109 (6) (2001) 2751-2761.

[6] A. Ramanathan, M Heilemann, and M. F. Bocko, Real-Time Multichannel Interfacing for a Dynamic Flat-Panel Audio Display Using the MATLAB Audio Systems Toolbox, in: Proceedings of the $143^{\text {rd }}$ Convention of Audio Engineering Society, 2017 
[7] J.-H. Woo, D.-Y. Kim, and J.-G. Ih, Virtual formation of a woofer at the roof panel of a vehicle by using array actuators, SAE Int. J. Passeng. Cars-Mech. Syst. 9 (3) (2016) 10861090.

[8] M. C. Heilemann, D. A. Anderson, S. Roessner, and M. F. Bocko, Quantifying Listener Preference of Flat-Panel Loudspeakers, in: Proceedings of the $145^{\text {th }}$ Convention of Audio Engineering Society, 2018, No. 10087.

[9] D. A. Anderson, M. C. Heilemann, and M. F. Bocko, Measures of vibrational localization on point-driven flat-panel loudspeakers, Proc. Mts. Acoust. 26, 065003 (2016).

[10] S. T. Christensen, S. V. Sorokin, and N. Olhoff, On analysis and optimization in structural acoustics - Part II: Exemplifications for axisymmetric structures, Struct. Optimization 16 (1998) pp. 96-107.

[11] S. T. Christensen, and N. Olhoff, Shape optimization of a loudspeaker diaphragm with respect to sound directivity properties, Control Cybern. 27(2) (1998) pp. 177-198.

[12] M. R. Bai and B. Liu, Determination of optimal exciter deployment for panel speakers using the genetic algorithm, J. Sound Vib. 269 (2004), pp. 727-743.

[13] D. A. Anderson, M. C. Heilemann, and M. F. Bocko, Optimized Driver Placement for Array-Driven Flat-Panel Loudspeakers, Arch. Acoust. 42(1) (2017), pp. 93-104.

[14] D. A. Anderson, M. C. Heilemann, and M. F. Bocko, Flat Panel Loudspeaker Simulation Model with Electromagnetic Inertial Exciters and Enclosures, J. Audio Eng. Soc. 65(9) (2017), pp. 722-732.

[15] G. Lu and Y. Shen, Model optimization of orthotropic distributed-mode loudspeaker using attached masse, J. Acoust. Soc. Am. 126 (5) (2009), pp. 2294-2300.

[16] G. Lu, Y. Shen, and Z. Liu, Optimization of orthotropic distributed-mode loudspeaker using attached masses and multi-exciters, J. Acoust. Soc. Am. 131, EL93 (2012).

[17] D. A. Anderson and M. F. Bocko, Modal Crossover Networks for Flat-Panel Loudspeakers, J. Audio Eng, Soc. 64(4) (2016) pp. 229-240.

[18] M. M. Boone, Multi-Actuator Panels (MAPs) as Loudspeaker Arrays for Wave Field Synthesis, J. Audio Eng. Soc. 52(7/8) (2004) pp. 712-723.

[19] J. H. Ho and A. P. Berkhoff, Flat acoustic sources with frequency response correction based on feedback and feed-forward distributed control, J. Acoust. Soc. Am. 137(4) (2015), pp. 2080-2088.

[20] M. C. Heilemann, D. A. Anderson, and M. F. Bocko, Sound-Source Localization on FlatPanel Loudspeakers, J. Audio Eng. Soc. 65(3) (2017) pp. 168-177. 
[21] Z. Li, C. Zheng, and X. Li, Vibrational Contrast Control for Local Sound Source Rendering on Flat Panel Loudspeakers, in: Proceedings of the $145^{\text {th }}$ Convention of Audio Engineering Society, 2018, No. 10086.

[22] L. Raghavan and A. Srikantha Phani, Local resonance bandgaps in periodic media: Theory and experiment, J. Acoust. Soc. Am. 134(3) (2013), pp. 1950-1959.

[23] C. Claeys, K. Vergote, P. Sas, and W. Desmet, On the potential of tuned resonators to obtain low-frequency vibrational stop bands in periodic panels, J. Sound and Vib. 332(6) (2013) pp. 1418-1436.

[24] A. Nateghi, L. Sangiuliano, C. Claeys, E Deckers, B. Pluymers, and W. Desmet, Design and experimental validation of a metamaterial solution for improved noise and vibration behavior of pipes, J. Sound and Vib. 455(1) (2019) pp. 96-117.

[25] N.G.R. de Melo Filho, L. Van Belle, C. Claeys, E. Deckers, and W. Desmet, Dynamic mass based sound transmission loss prediction of vibro-acoustic metamateiral double panels applied to the mass-air-mass resonance, J. Sound and Vib. 442(3) (2019) pp. 28-44.

[26] J. Jung, C.-H. Jeong, and J. S. Jensen, Efficient sound radiation using a bandgap structure, Appl. Phys. Lett. 115, 041903 (2019).

[27] J. Jung, H.-G. Kim, S. Goo, K.-J. Chang, and S. Wang, Realisation of a locally resonant metamaterial on the automobile panel structure to reduce noise radiation, Mech. Syst. Signal Process. 122, 206 (2019) pp. 206-231.

[28] N. M. M. Frandsen, O. R. Bilal, J. S. Jensen, and M. I. Hussein, Inertial amplification of continuous structures: Large band gaps from small masses, J. Appl. Phys. 119, 124902 (2016).

[29] C. Claeys, P. Sas, and W. Desmet, On the acoustic radiation efficiency of local resonance based stop band materials, J. Sound and Vib. 333(14) (2014) pp. 3203-3213.

[30] J. Henneberg, A. Gerlach, H. Storck, H. Cebulla, and S. Marburg, Reducing mechanical cross-coupling in phased array transducers using stop band material as backing, J. Sound and Vib. 424(23) (2018) pp. 352-364.

[31] J. Henneberg, A. Gerlach, H. Cebulla, and S. Marburg, The potential of stop band material in multi-frequency ultrasonic transducers, J. Sound and Vib. 452(21) (2019) pp. 132-146.

[32] K. J. Bathe, Finite Element Procedures, Prentice-Hall, New Jersey (1996)

[33] P. S. Lee, K. J. Bathe, Development of MITC isotropic triangular shell finite elements, Comput. Struct. 82(11-12) (2004) pp. 945-962. 
[34] W. Klippel and J. Schlechter, Distributed mechanical parameters of loudspeakers, part 2: Diagnostics, J. Audio Eng. Soc. 57(9) (2009) pp. 696-708.

[35] J. Jung, J. Kook, S. goo, and S. Wang, Sound transmission analysis of plate structures using the finite element method and elementary radiator approach with radiator error index, Adv. Eng. Softw. 112 (2017) pp. 1-15.

[36] D. Fritze, S. Marburg, H. J. Hardtke, Estimation of Radiated Sound Power- A Case study on Common Approximation Methods, Acta Acust. United Ac. 95(5) (2009) pp. 833-842.

[37] J. G. Tylka and E. Y. Choueiri, On the calculation of full and partial directivity indices 3D Audio and Applied Acoustics Laboratory Technical Report (Princeton University Press, Princeton, NJ, 2014)

[38] Dayton Audio, Exciter Buyers Guide. https://www.daytonaudio.com/index.php/excitersbuyers-guide, 2019 (accessed 19 August 2019).

[39] F. J. Fahy, P. Gardonio, Sound and Structural Vibration: Radiation, Transmission and Response $2^{\text {nd }}$ edition (Academic, Oxford, 2007). 